TRANSACTIONS OF THE

AMERICAN MATHEMATICAL SOCIETY

Volume 352, Number 10, Pages 4397-4419

S 0002-9947(00)02593-9

Article electronically published on February 24, 2000

\title{
DEFINABLY SIMPLE GROUPS IN O-MINIMAL STRUCTURES
}

\author{
Y. PETERZIL, A. PILLAY, AND S. STARCHENKO
}

\begin{abstract}
Let $\mathbb{G}=\langle G, \cdot\rangle$ be a group definable in an o-minimal structure $\mathcal{M}$. A subset $H$ of $G$ is $\mathbb{G}$-definable if $H$ is definable in the structure $\langle G, \cdot\rangle$ (while definable means definable in the structure $\mathcal{M}$ ). Assume $\mathbb{G}$ has no $\mathbb{G}$ definable proper subgroup of finite index. In this paper we prove that if $\mathbb{G}$ has no nontrivial abelian normal subgroup, then $\mathbb{G}$ is the direct product of $\mathbb{G}$-definable subgroups $H_{1}, \ldots, H_{k}$ such that each $H_{i}$ is definably isomorphic to a semialgebraic linear group over a definable real closed field. As a corollary we obtain an o-minimal analogue of Cherlin's conjecture.
\end{abstract}

This is the first of two papers around groups definable in o-minimal structures and semialgebraic groups over real closed fields.

An o-minimal structure is a structure $\mathcal{M}=\langle M,<, \ldots$.$\rangle where <$ is a dense linear ordering of $M$, and any definable subset of $M$ is a finite union of intervals (with endpoints in $M \cup\{ \pm \infty\}$ ) and points. A group $G$ is said to be definable in $\mathcal{M}$ if both $G$ and the graph of the group operation on $G$ are definable sets in $\mathcal{M}$ (i.e. definable subsets of $M^{n}, M^{3 n}$ for some $n$ ). The typical example is $G=H(\mathcal{R})$ where $H$ is an algebraic group defined over a real closed field $\mathcal{R}$. (Take $\mathcal{M}=\langle\mathcal{R},<,+, \cdot$,$\rangle .)$ We show a converse: suppose that $G$ is definable in some o-minimal structure and that $G$ is nonabelian and has no proper nontrivial normal subgroup definable in the structure $\langle G, \cdot\rangle$ (we say that $G$ is $\mathbb{G}$-definably simple). Then $G$ is isomorphic to an (open) semialgebraic subgroup of finite index of a group of the form $H(\mathcal{R})$, where $\mathcal{R}$ is a real closed field and $H$ is an $\mathcal{R}$-simple algebraic group. This gives a positive answer to the o-minimal analogue of the (yet unproved) Cherlin-Zilber conjecture: any simple group of finite Morley rank is an algebraic group over an algebraically closed field.

The strategy of our proof is closely related to Poizat's approach ([12]) to Cherlin's conjecture. Given $G$ definable in o-minimal $\mathcal{M}$, we try to find a real closed field $\mathcal{R}$ definable in $\mathcal{M}$ which is intimately connected to $G$. We then try to show that $G$ is definably (in $\mathcal{M}$ ) isomorphic to a linear semialgebraic group over $\mathcal{R}$. The first step is made possible by, among other things, the Trichotomy theorem. The second step goes through developing Lie theory over o-minimal expansions of real closed fields. This second step is possible, because, once we have a real closed field $\mathcal{R}$ definable in an o-minimal structure $\mathcal{M}$, then definable (in $\mathcal{M}$ ) functions on $\mathcal{R}^{n}$ are piecewise as differentiable as one wants.

In practice it is convenient to work with centerless and "semisimple" groups, namely groups with no nontrivial normal abelian subgroups, and for these we prove

Received by the editors February 25, 1998.

2000 Mathematics Subject Classification. Primary 03C64, 22E15, 20G20; Secondary 12J15.

The second and the third authors were partially supported by NSF. 
the theorem stated in the abstract. The formal definition of o-minimality allows several non-definably isomorphic real closed fields to live on a single o-minimal structure; for example, let $\mathcal{M}$ be the structure on a dense linear ordering formed by putting nonisomorphic real closed fields on two disjoint open intervals $(a, b)$ and $(c, d)$. This presents various technical problems, which are dealt with in several parts of this paper.

In the second paper $([9])$ we begin with the end result of this paper: given $G$ a semialgebraic group over a real closed field $\mathcal{R}$, and assuming that $G$ is $\mathbb{G}$ definably simple, we show that $\langle G, \cdot\rangle$ is "bi-interpretable" with the field $\mathcal{R}$ or with the algebraically closed field $\mathcal{R}(i)$. This says, in particular, that if $G$ is a simple real Lie group, then the abstract group $G$ is "identical" in a definite model-theoretic sense with either the real field or with the complex field. This automatically yields various (well-known) rigidity theorems for simple real Lie groups.

We would like to thank the referee for the careful reading of the paper and for many valuable comments and suggestions.

The structure of the paper. In Section 1 we establish some properties of definable differentiable manifolds and their tangent spaces; prove technical lemmas about groups definable in o-minimal structures; and develop basics of nonorthogonality.

In Section 2 we deal with groups definable in o-minimal expansions of real closed fields. After establishing basic properties of the Lie correspondence, we prove the main result for such groups.

In Section 3 we show that if a group $G$ is $\mathbb{G}$-definably connected, centerless and is definable in an o-minimal structure $\mathcal{M}$, then $G$ is the direct product of $\mathbb{G}$ definable subgroups $H_{1}, \ldots, H_{k}$, such that each $H_{i}$ is definably isomorphic to a group definable in an o-minimal expansion of a real closed field.

In Section 4 combining results of Sections 2 and 3 we will prove the main theorem.

After the paper was submitted the authors realized that the main result of this paper, together with Strzebonski's analysis of o-minimal abelian groups, yields the positive answer to the following conjecture of Strzebonski [13]: If $G$ is a definably connected group definable in an o-minimal structure, then the Euler characteristic of $G$ is either 0 or \pm 1 .

\section{Preliminaries}

We will assume that the reader is familiar with basic facts about o-minimal structures. (They can be found in 11, 8, 4]). A good source of definitions and properties of dimension and generic points is [11.

Throughout this paper $\mathcal{M}=\langle M,<, \ldots\rangle$ will be an o-minimal structure. $M$ is equipped with the interval topology and Cartesian powers $M^{n}$ with product topologies. Unless otherwise stated, we will refer to these topologies.

Very often we will not distinguish between tuples and elements. So the notation $a \in M^{k}$ is legitimate.

We say that $a$ set $D \subseteq M^{k}$ is definable over a set $A \subseteq M$ if there is a first order formula $\varphi(x)$ with parameters from $A$ such that $D=\left\{d \in M^{k} \mid \mathcal{M} \models \varphi(d)\right\}$. A set $D \subseteq M^{k}$ is definable if it is definable over $M$. A function $f: D \subseteq M^{k} \rightarrow M^{n}$ is definable if its graph is a definable set. A group $\langle G, \cdot, \mathbf{e}\rangle$ is definable if $G$ is a definable set and the group multiplication is a definable function. Similarly, a field 
$\mathcal{R}$ is definable in $\mathcal{M}$ if its universe is a definable set and the field operations are definable functions.

Usually, we will denote a group $\langle G, \cdot, \mathbf{e}\rangle$ by the same letter $G$ as its universe. However, if we need to explicitly refer to the first order structure of a group, we will use letters $\mathbb{G}, \mathbb{H}$, etc., for them.

If $\langle G, \cdot, \mathbf{e}\rangle$ is a group definable in $\mathcal{M}$, then a subset $A \subseteq G^{k}$ is called $\mathbb{G}$-definable if it is definable in the structure $\mathbb{G}$ (i.e., it is definable in the group language), otherwise, definable means definable in $\mathcal{M}$.

A definable group $G$ is $\mathbb{G}$-definably simple if it is infinite, not abelian and does not contain a $\mathbb{G}$-definable proper nontrivial normal subgroup.

A definable group $G$ is $\mathbb{G}$-definably connected if it has no proper $\mathbb{G}$-definable subgroup of finite index.

Recall that if $\mathcal{M}$ is an o-minimal expansion of a real closed field $\mathcal{R}$, then a definable subset $A \subseteq M^{k}$ is semialgebraic if it is definable by a formula (with parameters from $M$ ) in the field language.

If $\mathcal{M}$ is an expansion of a real closed field $\mathcal{R}$, then the usual definition of derivative makes sense there; and we say that a function $f$ from an open set $D \subseteq M^{n}$ into $M^{l}$ is of class $C^{p}$ on $D$ if its $k$-th derivative exists for $1 \leq k \leq p$, and is continuous. Also basic differentiable calculus is true for functions definable in such structures (see [1] for details).

Let $X$ be a definable set. Recall that a definable subset $Y$ of $X$ is called large in $X$ if $\operatorname{dim}(X \backslash Y)<\operatorname{dim}(X)$.

We will use extensively the following fact.

Fact 1.1. Let $D$ be a subset of $M^{k}$ with $\operatorname{dim}(D)=k$ and let $f: D \rightarrow M^{n}$ be a definable function. Assume that both $D$ and $f$ are definable over a set $A$. Then there is a definable over $A$ large subset $S$ of $D$ such that $S$ is open in $M^{k}$ and $f$ is continuous on $S$. In addition, if $\mathcal{M}$ is an expansion of a real closed field, then for any $p \geq 0, S$ can be chosen so that $f$ is $C^{p}$ on $S$.

The following fact will be needed in Section 1.1.2.

Fact 1.2. Suppose $\mathcal{M}$ is an o-minimal expansion of a real closed field and $D$ is an open subset of $M^{k}$. If $f: D \rightarrow M^{n}$ is a definable $C^{1}$-function with $k=\operatorname{dim}(f(D))$, then there is $d \in D$ such that the rank of the differential of $f$ at $d$ is equal to $k$.

1.1. Definable manifolds over o-minimal structures. The notion of a definable manifold over an o-minimal structure was introduced in 11. In this paper we are interested mostly in definable differentiable manifolds and their tangent spaces. In the case of o-minimal expansions of the field of the real numbers, such manifolds were extensively studied in [2].

We fix an integer $p \geq 0$, and we will always assume that if $p>0$, then $\mathcal{M}$ is an expansion of a real closed field.

Let $X$ be a definable set.

A definable chart on $X$ is a triple $\mathfrak{c}=\langle U, \varphi, n\rangle$, where $U$ is a definable subset of $X, n \geq 0$ and $\varphi$ is a definable bijection from $U$ onto an open subset of $M^{n}$. If $\mathfrak{c}=\langle U, \varphi, n\rangle$ is a definable chart, then $n$ is called the dimension of $\mathfrak{c}$.

Two definable charts $\mathfrak{c}=\langle U, \varphi, n\rangle$ and $\mathfrak{c}^{\prime}=\left\langle U^{\prime}, \varphi^{\prime}, n^{\prime}\right\rangle$ are $C^{p}$-compatible if either $U \cap U^{\prime}=\varnothing$ or $\varphi\left(U \cap U^{\prime}\right)$ is open, $\varphi^{\prime}\left(U^{\prime} \cap U\right)$ is open, and the two transition mappings $\varphi \circ \varphi^{\prime-1}$ and $\varphi^{\prime} \circ \varphi^{-1}$ are of class $C^{p}$ on their domains; note that in the last case $n=n^{\prime}$. 
$A$ definable $C^{p}$-atlas on $X$ is a finite set $\mathfrak{C}$ of definable charts on $X$, each pair of which is $C^{p}$-compatible and whose domains cover $X$.

$A$ definable $C^{p}$-manifold $\mathbf{X}$ is a pair $\langle X, \mathfrak{C}\rangle$, where $X$ is a definable set and $\mathfrak{C}$ is a definable $C^{p}$-atlas on $X$.

If $\mathbf{X}=\langle X, \mathfrak{C}\rangle$ is a definable $C^{p}$-manifold, and $\mathfrak{c}$ is a definable chart on $X$, then we say that $\mathfrak{c}$ is a definable $C^{p}$-chart on $\mathbf{X}$ if $\mathfrak{C} \cup\{\mathfrak{c}\}$ is a definable $C^{p}$-atlas, i.e. $\mathfrak{c}$ is $C^{p}$-compatible with every chart in $\mathfrak{C}$.

We say that two definable $C^{p}$-manifolds $\left\langle X_{1}, \mathfrak{C}_{1}\right\rangle$ and $\left\langle X_{2}, \mathfrak{C}_{2}\right\rangle$ are equal if $X_{1}=$ $X_{2}$ and $\mathfrak{C}_{1} \cup \mathfrak{C}_{2}$ is a definable $C^{p}$-atlas on $X_{1}$.

We will use bold letters $\mathbf{X}, \mathbf{Y}, \ldots$ to denote definable manifolds whose underlying sets are $X, Y, \ldots$ If for a definable $C^{p}$-manifold $\mathbf{X}=\langle X, \mathfrak{C}\rangle$ we want to specify that $X$ and $\mathfrak{C}$ are definable in the structure $\mathcal{M}$, then we say that $\mathbf{X}$ is a definable $C^{p}$-manifold over $\mathcal{M}$.

Let $\mathfrak{c}=\langle U, \varphi, n\rangle$ be a definable chart on a definable $C^{p}$-manifold $\mathbf{X}$. For $a \in U$ we say that $\mathfrak{c}$ is a chart on $\mathbf{X}$ at $a$; and call $n$ the dimension of $\mathbf{X}$ at $a$.

Let $\mathbf{X}$ and $\mathbf{Y}$ be definable $C^{p}$-manifolds, $f: X \rightarrow Y$ a function and $a \in X$. For $k \leq p$, we say that $f$ is of class $C^{k}$ at $a$ if, for any charts $\langle U, \varphi, n\rangle$ on $\mathbf{X}$ at $a$ and $\left\langle V, \psi, n^{\prime}\right\rangle$ on $\mathbf{Y}$ at $f(a)$, the mapping

$$
F=\psi \circ f \circ \varphi^{-1}: \varphi(U) \rightarrow \psi(V)
$$

is of class $C^{k}$. (Since all transition maps are $C^{p}$, we can replace in the above definition "for any charts" by "for some charts".) We say that $f$ is of class $C^{k}$ on $\mathbf{X}$ if it is $C^{k}$ at every $a \in X$.

If $\mathbf{X}$ is a definable $C^{0}$-manifold, then we can equip $X$ with a unique topology $\tau$ so that, for any definable chart $\langle U, \varphi, n\rangle$ on $\mathbf{X}$, a subset $V$ of $U$ is open in $\tau$ if and only if $\varphi(V)$ is an open subset of $M^{n}$. We will refer to this topology $\tau$ as the manifold topology of $\mathbf{X}$.

1.1.1. Definable connectedness. A definable subset $S$ of $M^{n}$ is definably connected if there are no definable open (in the product topology of $\mathcal{M}$ ) disjoint sets $U_{1}, U_{2} \subseteq$ $M^{n}$ such that $S \subseteq U_{1} \cup U_{2}$ and $S \cap U_{i} \neq \varnothing, i=1,2$.

Let $\mathbf{X}$ be a definable $C^{0}$-manifold. A definable subset $S$ of $X$ is definably connected in $\mathbf{X}$ if there are no nonempty open (in the manifold topology) disjoint definable sets $U_{1}, U_{2} \subseteq X$ such that $S \subseteq U_{1} \cup U_{2}$ and $S \cap U_{i} \neq \varnothing, i=1,2$. A definable $C^{0}$-manifold $\mathbf{X}$ is definably connected if its underlying set $X$ is definably connected in $\mathbf{X}$.

Claim 1.3. Let $\mathcal{N}$ be an o-minimal expansion of $\mathcal{M}$.

1. If $U$ is an open subset of $N^{n}$, definable in $\mathcal{M}$, then there are unique definable, open, definably connected in $\mathcal{N}$ sets $V_{1}, \ldots, V_{m} \subseteq U$ such that $U$ is the disjoint union of $V_{1}, \ldots, V_{m}$. Moreover, all $V_{i}$ are definable in $\mathcal{M}$.

2. If $\mathbf{X}$ is a definable $C^{0}$-manifold over $\mathcal{N}$, then there are unique definable, open, definably connected in $\mathbf{X}$ sets $V_{1}, \ldots, V_{m} \subseteq X$ such that $X$ is the disjoint union of $V_{1}, \ldots, V_{m}$. Moreover, if $\mathbf{X}$ is definable in $\mathcal{M}$, then all $V_{i}$ are definable in $\mathcal{M}$.

Proof. By standard arguments, uniqueness follows easily from the existence of such $V_{i}$.

First, note that every cell definable in $\mathcal{N}$ is definably connected in $\mathcal{N}$.

1. By cell decomposition, $U$ is a finite union of cells $C_{1}, \ldots, C_{k}$ that are definable in $\mathcal{M}$, since $U$ is definable in $\mathcal{M}$. We will prove the statement by induction on $k$. 
If $k=1$, then $U$ is a cell, and it is definably connected in $\mathcal{N}$.

Induction step. Suppose $U$ is not definably connected in $\mathcal{N}$. Then there are nonempty open definable in $\mathcal{N}$ sets $U_{1}, U_{2} \subseteq U$ such that $U$ is the disjoint union of $U_{1}$ and $U_{2}$. Since every cell is definably connected in $\mathcal{N}$, for every $i \in\{1, \cdots, k\}$ either $C_{i} \subseteq U_{1}$ or $C_{i} \subseteq U_{2}$. So the cells $C_{i}$, such that $C_{i} \subseteq U_{1}$, cover $U_{1}$. Hence, since all $C_{i}$ are $\mathcal{M}$-definable, $U_{1}$ is $\mathcal{M}$-definable, and, by the induction hypotheses, $U_{1}$ is the disjoint union of open definably connected in $\mathcal{N}$ sets which are definable in $\mathcal{M}$. The same is true for $U_{2}$, and therefore for $U$.

2. We assume that $\mathbf{X}=\langle X, \mathfrak{C}\rangle$ is a manifold over $\mathcal{M}$. Applying cell decomposition to sets $\varphi(U)$ for every chart $\langle U, \varphi, n\rangle \in \mathfrak{C}$, we obtain a finite collection of definable in $\mathcal{M}$ sets $C_{1}, \ldots, C_{k}$ such that $X=\bigcup_{i=1}^{k} C_{i}$ and for every $C_{i}$ there is a chart $\langle U, \varphi, n\rangle \in \mathfrak{C}$ such that $\varphi\left(C_{i}\right)$ is a cell. The rest of the proof is as in 1.

Let $\mathbf{X}$ be a definable manifold over $\mathcal{M}$. The definably connected sets $V_{1} \ldots, V_{k}$ from the previous claim will be called the definably connected components of $\mathbf{X}$. By the same claim, if $\mathcal{N}$ is an o-minimal expansion of $\mathcal{M}$, then definably connected components of $\mathbf{X}$ in $\mathcal{N}$ are the same as in $\mathcal{M}$. For example, if $\mathcal{M}$ is an expansion of a real closed field $\mathcal{R}$, and $U$ is an open semialgebraic subset of $M^{n}$, then the definably connected components of $U$ in $\mathcal{M}$ are semialgebraic.

1.1.2. Differentiable manifolds and their tangent spaces. In this section $\mathcal{M}$ will denote an o-minimal expansion of a real closed field $\mathcal{R}$. We fix an integer $p>0$.

Let $\mathbf{X}, \mathbf{Y}$ be definable $C^{p}$-manifolds, and let $f, g: \mathbf{X} \rightarrow \mathbf{Y}$ be definable $C^{1}$ functions. We say that $f$ and $g$ are tangent at $a \in X$ if $f(a)=g(a)$ and for any charts $\langle U, \varphi, n\rangle$ on $\mathbf{X}$ at $a$ and $\left\langle V, \psi, n^{\prime}\right\rangle$ on $\mathbf{Y}$ at $f(a)$ the mappings $\psi \circ f \circ \varphi^{-1}$ and $\psi \circ g \circ \varphi^{-1}$ have the same differential at $\varphi(a)$. Once again, we can replace in the above definition "for any charts" by "for some charts".

Claim 1.4. Let $\mathbf{X}, \mathbf{Y}$, and $\mathbf{Z}$ be definable $C^{p}$-manifolds, $f_{1}, f_{2}: \mathbf{X} \rightarrow \mathbf{Y}$ and $g: \mathbf{Y} \rightarrow \mathbf{Z}$ definable $C^{1}$-mappings, and $x \in X$. If $f_{1}$ and $f_{2}$ are tangent at $x$, then $g \circ f_{1}$ is tangent to $g \circ f_{2}$ at $x$.

Proof. Follows from the Chain Rule.

Let $\mathbf{X}$ be a definable $C^{p}$-manifold and $x \in X$. Consider the relation " $f_{1}$ and $f_{2}$ are tangent at 0 " on the set of all definable $C^{1}$-functions $f$ from $\mathcal{R}$ into $\mathbf{X}$ with $f(0)=x$.

Claim 1.5. The relation " $f_{1}$ and $f_{2}$ are tangent at 0 " is an equivalence relation.

Proof. Easy.

The equivalence classes for this relation are called the tangent vectors to $\mathbf{X}$ at $x$, and the set of all classes is denoted by $\mathcal{T}_{x}(\mathbf{X})$.

For every definable chart $\mathfrak{c}=\langle U, \varphi, n\rangle$ on $\mathbf{X}$ at $x$ there is a canonical bijection $\Theta_{\mathfrak{c}}: \mathcal{T}_{x}(\mathbf{X}) \rightarrow \mathcal{R}^{n}$ that maps the class of a function $f: \mathcal{R} \rightarrow \mathbf{X}$ to the vector $\mathrm{d}(\varphi \circ f)(0)$. Thus we can use $\Theta_{\mathfrak{c}}^{-1}$ to transpose the vector space structure of $\mathcal{R}^{n}$ onto $\mathcal{T}_{x}(\mathbf{X})$. If $\mathfrak{c}^{\prime}=\left\langle U^{\prime}, \varphi^{\prime}, n\right\rangle$ is another chart, then the mapping $\Theta_{\mathfrak{c}^{\prime}} \circ \Theta_{\mathfrak{c}}^{-1}$ is a bijective linear mapping, and therefore this vector space structure on $\mathcal{T}_{x}(\mathbf{X})$ does not depend on the choice of $\boldsymbol{c}$.

The set $\mathcal{T}_{x}(\mathbf{X})$, endowed with this vector space structure, is called the tangent vector space (or, simply, the tangent space) of $\mathbf{X}$ at $x$. 
Notice that $\mathcal{T}_{x}(\mathbf{X})$, defined this way, is not a definable object. However, after fixing a chart $\mathfrak{c}$, we can identify, by virtue of $\Theta_{\mathfrak{c}}, \mathcal{T}_{x}(\mathbf{X})$ with the vector space $\mathcal{R}^{n}$.

If $\mathbf{Y}, \mathbf{X}$ are definable $C^{p}$-manifolds, $m \in X$, and $f: \mathbf{X} \rightarrow \mathbf{Y}$ is a definable $C^{1}$-mapping, then, by Claim [1.4 $f$ induces a mapping from $\mathcal{T}_{m}(\mathbf{X})$ into $\mathcal{T}_{f(m)}(\mathbf{Y})$. We will denote this mapping by $\mathrm{d}_{m}(f)$. Obviously, $\mathrm{d}_{m}(f)$ is linear. Also, if $\mathfrak{c}=$ $\langle U, \varphi, n\rangle$ is a chart on $\mathbf{X}$ at $m$ and $\mathfrak{c}^{\prime}=\langle V, \psi, k\rangle$ is a chart on $\mathbf{Y}$ at $f(m)$, then the linear mapping $\Theta_{\mathfrak{c}^{\prime}} \circ \mathrm{d}_{m}(f) \circ \Theta_{\mathfrak{c}}^{-1}$ is equal to the differential of $\psi \circ f \circ \varphi^{-1}$ at $\varphi(m)$.

Claim 1.6. If $f$ is a definable $C^{1}$-mapping from a definable $C^{p}$-manifold $\mathbf{X}$ into a definable $C^{p}$-manifold $\mathbf{Y}$, then there is $m \in X$ such that the rank of $\mathrm{d}_{m}(f)$ is equal to the dimension of $f(X)$ at $f(m)$.

Proof. Follows from Fact 1.2 after choosing appropriate charts on $\mathbf{X}$ and $\mathbf{Y}$.

Claim 1.7. If $\mathbf{X}$ is a definably connected definable $C^{p}$-manifold, and $f$ is a definable $C^{1}$-function from $\mathbf{X}$ into a definable $C^{p}$-manifold $\mathbf{Y}$ such that $\mathrm{d}_{x}(f)=0$ for any $x \in X$, then $f$ is a constant function.

Proof. Easy.

Claim 1.8. If $f$ is a definable injective $C^{1}$-function from a definable $C^{p}$-manifold $\mathbf{X}$ into a definable $C^{p}$-manifold $\mathbf{Y}$, then there is $x \in X$ such that $\mathrm{d}_{x}(f)$ is injective.

Proof. By Claim [1.6 there is $x \in X$ such that the rank of $\mathrm{d}_{x}(f)$ is equal to the dimension of $f(X)$ at $f(x)$. Let $k$ be the rank of $\mathrm{d}_{x}(f)$. Since $f$ is injective the dimension of $X$ at $x$ cannot be greater than $k$, and therefore the kernel of $\mathrm{d}_{x}(f)$ must be 0 .

A definable $C^{p}$-mapping $f$ from a definable $C^{p}$-manifold $\mathbf{X}$ into a definable $C^{p}$ manifold $\mathbf{Y}$ is called an immersion if for any $y \in X$ the mapping $\mathrm{d}_{y}(f)$ is injective.

Definition 1.9. Let $\mathbf{X}$ be a definable $C^{p}$-manifold.

1. A definable $C^{p}$-manifold $\mathbf{Y}$ is a submanifold of $\mathbf{X}$ if $Y \subseteq X$ and $\operatorname{id}_{Y}: \mathbf{Y} \rightarrow \mathbf{X}$ is an immersion.

2. A definable subset $Y$ of $X$ is a submanifold of $\mathbf{X}$ if $Y$ possess a definable $C^{p}$-manifold structure $\mathbf{Y}$ such that $\mathbf{Y}$ is a submanifold of $\mathbf{X}$.

Note. If $\mathbf{Y}$ is a submanifold of $\mathbf{X}$ and $y \in Y$, then $\mathrm{d}_{y}\left(\operatorname{id}_{Y}\right)$ is injective, and we can always consider $\mathcal{T}_{y}(\mathbf{Y})$ as a subspace of $\mathcal{T}_{y}(\mathbf{X})$.

1.2. Groups definable in o-minimal structures. In this section we present some basic facts about groups definable in o-minimal structures. Most of them can be found in [11].

We fix an integer $p \geq 0$, and, as usual, if $p>0$, then $\mathcal{M}$ is assumed to be an expansion of a real closed field.

Let $G$ be a group definable in $\mathcal{M}$. A pair $\langle G, \mathfrak{A}\rangle$ is a definable $C^{p}$-group if $\mathfrak{A}$ is a definable $C^{p}$-atlas on $G$ and group multiplication and inversion are $C^{p}$-mappings.

Note that if $\langle G, \mathfrak{A}\rangle$ is a definable $C^{0}$-group, then for all $a, b \in G$ the function $x \mapsto x a^{-1} b$ is a homeomorphism that maps $a$ into $b$. Therefore the dimension of $\langle G, \mathfrak{A}\rangle$ at any point is the same and equals $\operatorname{dim}(G)$.

Fact 1.10. 11] If $G$ is a group definable in $\mathcal{M}$, then there is an atlas $\mathfrak{A}$ on $G$ such that $\langle G, \mathfrak{A}\rangle$ is a definable $C^{p}$-group. 
Proof. If $p=0$, then this is a restatement of Proposition 2.5 in [11. If $p>0$, then the same proof works when one replaces "continuous" by " $C$ " ".

Lemma 1.11. Let $\langle G, \mathfrak{A}\rangle$, and $\langle H, \mathfrak{B}\rangle$ be definable $C^{p}$-groups. If $f: H \rightarrow G$ is a definable group homomorphism, then $f$ is $C^{p}$.

Proof. Since $f$ is a homomorphism and the group operations are $C^{p}$, by the Chain Rule, it is sufficient to show that $f$ is $C^{p}$ at one point. Let $\left\langle U_{i}, \varphi_{i}, n\right\rangle, i=1, \ldots, k$, be a list of all charts in $\mathfrak{A}$, and $\langle V, \psi, m\rangle$ be a chart in $\mathfrak{B}$.

Since $V \subseteq \bigcup_{1 \leq i \leq k} f^{-1}\left(U_{i}\right)$, there is $j \in\{1, \ldots, k\}$ such that

$$
\operatorname{dim}\left(\psi\left(f^{-1}\left(U_{j}\right)\right) \cap \psi(V)\right)=\operatorname{dim}(\psi(V)) .
$$

Thus there is an open definable $V_{0} \subseteq V$ such that $f\left(V_{0}\right) \subseteq U_{j}$. By Fact 1.1, there is $c \in V_{0}$ such that the function $\varphi \circ f \circ \psi^{-1}$ is $C^{p}$ at $\psi(c)$, and therefore $f$ is $C^{p}$ at $c$.

Corollary 1.12. If $G$ is a definable group, then the definable $C^{p}$-group structure on $G$ is unique.

Proof. Let $\mathfrak{A}$ and $\mathfrak{B}$ be two definable atlases on $G$ such that both $\mathbf{G}_{1}=\langle\mathbb{G}, \mathfrak{A}\rangle$ and $\mathbf{G}_{2}=\langle\mathbb{G}, \mathfrak{B}\rangle$ are definable $C^{p}$-groups. Applying Lemma 1.11 to the identity mapping id : $G \rightarrow G$ we obtain that every chart in $\mathfrak{A}$ is $C^{p}$-compatible with every chart in $\mathfrak{B}$.

If $G$ is a definable group, then it possesses a unique definable $C^{0}$-manifold structure that makes it a definable $C^{0}$-group. We will use the same letters $G, H, \ldots$, to denote the definable $C^{0}$-manifold structures on $G, H, \ldots$, and refer to the manifold topology of a definable group $G$ as the topology of $G$.

Until the end of this section $G$ will be a group definable in $\mathcal{M}$.

Fact 1.13 ([11]). G satisfies the descending chain condition on definable subgroups.

Fact 1.14 ([11]). For a definable subgroup $H$ of $G$ the following conditions are equivalent.

1. $H$ has a finite index in $G$.

2. $\operatorname{dim}(H)=\operatorname{dim}(G)$.

3. $H$ contains an open neighborhood of $\mathbf{e}$.

4. $H$ is open in $G$.

We will denote by $G^{0}$ the definably connected component of $\mathbf{e}$ in $G$.

Fact 1.15 ([1]). $G^{0}$ is the smallest definable subgroup of $G$ of a finite index.

Corollary 1.16. Let $S$ be a subset of $G$ (definable or not). Then $\mathbf{C}_{G}(S)$ $=\{g \in G \mid g s=s g$ for all $s \in S\}$, the centralizer of $S$ in $G$, is a $\mathbb{G}$-definable subgroup. (In fact, there is finite $S_{0} \subseteq S$ such that $\mathbf{C}_{G}(S)=\mathbf{C}_{G}\left(S_{0}\right)$ ).

Proof. Follows from Fact 1.13

Corollary 1.17. If $A$ is an abelian subgroup of $G$ (definable or not), then the center of $\mathbf{C}_{G}(A)$ is a $\mathbb{G}$-definable abelian subgroup containing $A$, which is normal if $A$ is normal.

Corollary 1.18. Assume $G$ is centerless. If $G$ is the direct product of subgroups $H_{1}$ and $H_{2}$, then both $H_{1}$ and $H_{2}$ are $\mathbb{G}$-definable.

Proof. It can be easily checked that $H_{1}=\mathbf{C}_{G}\left(H_{2}\right)$ and $H_{2}=\mathbf{C}_{G}\left(H_{1}\right)$. 
1.3. Nonorthogonality. In this section we establish some basic properties of nonorthogonality for intervals and prove some technical lemmas that will be used in Section 3 .

An open interval $I \subseteq M$ is transitive if for all $a, b \in I$ there are open definably homeomorphic subintervals $O_{a}, O_{b}$ of $I$, containing $a$ and $b$ respectively, and a definable homeomorphism $f: O_{a} \rightarrow O_{b}$ with $f(a)=b$.

An open rectangular box $I_{1} \times \ldots \times I_{n}$ is transitive if all intervals $I_{k}$ are transitive.

Two open transitive intervals $I, J \subseteq M$ are nonorthogonal if there is a definable homeomorphism between some open subintervals $I_{0} \subseteq I$ and $J_{0} \subseteq J$.

Two open transitive intervals $I, J \subseteq M$ are orthogonal if they are not nonorthogonal.

Two open rectangular transitive boxes $I_{1} \times \ldots \times I_{k}$ and $J_{1} \times \ldots \times J_{s}$ are orthogonal if each $I_{i}, i=1, \ldots, k$, is orthogonal to every $J_{j}, j=1, \ldots, s$.

Claim 1.19. Let $B_{1}, B_{2}$ be two orthogonal open transitive rectangular boxes. If $U$ is an open rectangular subset of $B_{1}$, then $U$ is orthogonal to $B_{2}$.

Proof. Easy.

Claim 1.20. 1. Let $J, J_{1}, \ldots, J_{s}$ be transitive open intervals with $J$ orthogonal to every $J_{i}, i=1, \ldots, s$. If $f$ is a definable continuous function from $J_{1} \times$ $\cdots \times J_{s}$ into $J$, then $f$ is a constant function.

2. Let $B_{1}, B_{2}$ be open rectangular transitive orthogonal boxes. If $f$ is a definable continuous function from $B_{1}$ into $B_{2}$, then $f$ is a constant function.

Proof. Obviously, 2 follows from 1.

To prove 1 it is sufficient to show that for any $i \in\{1, \ldots, s\}$ and any $\left\langle a_{1}, \ldots, a_{s}\right\rangle$ $\in J_{1} \times \cdots \times J_{s}$ the function

$$
f^{i}: x \mapsto f\left(a_{1}, \ldots, a_{i-1}, x, a_{i+1}, \ldots, a_{s}\right)
$$

is constant on $J_{i}$. If not then, by Monotonicity Theorem, there would be a subinterval $I \subseteq J_{i}$ such that $f^{i}$ was strictly monotone on $I$, so $f^{i}$ would be a homeomorphism from $I$ into a subinterval of $J$.

The following definition and fact can be found in [5].

Definition 1.21. An element $a \in M$ is nontrivial if there is an open interval $I$ containing $a$, an open interval $J$ and a definable continuous function $F: I \times J \rightarrow M$ which is 1-1 in each argument.

Fact 1.22. An element $a \in M$ is nontrivial if and only if there is an open transitive interval I containing a.

The following definition is taken from Stability Theory.

Definition 1.23. We say that a set $B=\left\{a_{1}, a_{2}, a_{3}\right\}$ is a triangle over a set $A$ if $a_{i} \in \operatorname{acl}\left(A \cup\left(B \backslash\left\{a_{i}\right\}\right)\right)$, for $i=1,2,3$, and $a_{i}$ is not in $\operatorname{acl}\left(A \cup\left\{a_{j}\right\}\right)$, for $i \neq j$.

Claim 1.24. If $c$ is a part of a triangle over a set $A$, then $c$ is nontrivial.

Proof. Easy.

Claim 1.25. Let $A$ and $B=\left\{b_{1}, \ldots b_{k}\right\}$ be subsets of $M$. If $a \in \operatorname{acl}(A B)$ and $a$ is not in $\operatorname{acl}\left(A \cup\left\{b_{i}\right\}\right)$ for any $b_{i} \in B$, then a is nontrivial. 
Proof. By induction on $k$.

If $k=2$, then it can be easily checked that $\left\{a, b_{1}, b_{2}\right\}$ is a triangle over $A$, and we are done by Claim 1.24

If $k>2$, then it suffices to show that we can decrease $B$, probably increasing $A$, preserving the conditions of the claim. If $a \in \operatorname{acl}\left(A, b_{1}, b_{k}\right)$, for some $b_{k} \in B$, then $a, b_{1}, b_{k}$ form a triangle, and we are done. If not, then $A \cup\left\{b_{1}\right\},\left\{b_{2}, \ldots, b_{k}\right\}$ and $a$ satisfy the conditions of the claim.

An open rectangle $B=I_{1} \times \ldots \times I_{k}$ is unidimensional if all intervals $I_{j}$ are transitive and pairwise nonorthogonal.

A definable in $\mathcal{M}$ group $G$ is unidimensional if there is a definable chart $\langle U, \varphi, n\rangle$ on $G$ at e such that $\varphi(U)$ is a unidimensional set.

Claim 1.26. $\quad 1$. If $B$ is a unidimensional open rectangle of dimension $k$, then there is an open transitive interval $I$ and definable continuous injection $f$ : $I^{k} \rightarrow B$.

2. If $G$ is a definable unidimensional group, then there is a definable chart $\langle U, \varphi, n\rangle$ on $G$ at $\mathbf{e}$ such that $\varphi(U)$ has the form $I^{n}$ for some open transitive interval $I$, and $\varphi(\mathbf{e})=\langle d, \ldots, d\rangle$ for some $d \in I$.

Proof. Easy.

Lemma 1.27. Let $G$ be a group definable in $\mathcal{M}$, and let $\mathfrak{c}=\langle U, \varphi, n\rangle$ be a definable chart on $G$ at $\mathbf{e}$. Then there is an element $g \in U$ such that for $\left\langle a_{1}, \ldots, a_{n}\right\rangle=\varphi(g)$ every $a_{i}$ is nontrivial.

Proof. Since, by Fact 1.22 , the nontriviality of any element can be witnessed by a formula, it is sufficient to show the existence of such $g$ in an $\omega$-saturated extension of $\mathcal{M}$. Without loss of generality we can assume $\mathcal{M}$ is $\omega$-saturated. We will also assume that $G$, the group multiplication, and the chart $\mathfrak{c}$ are definable over the empty set. Since the group multiplication is continuous on $U$, we can find independent generic elements $m, h \in U$ such that $g=m h$ is in $U$. Let $a=\left\langle a_{1}, \ldots, a_{n}\right\rangle=\varphi(g)$.

Since $g, m$ and $h$ are pairwise independent, we have

$$
\operatorname{dim}(\varphi(g) / m)=\operatorname{dim}(\varphi(g) / h)=n .
$$

Thus $a_{i} \notin \operatorname{acl}(m)$ and $a_{i} \notin \operatorname{acl}(h)$ for any $i \in\{1, \ldots, n\}$. As $a \in \operatorname{acl}(m, h)$, by Lemma 1.25, every $a_{i}$ is nontrivial.

Lemma 1.28. Let $G$ be a group definable in $\mathcal{M}$. Then there is a definable chart $=\langle U, \psi, n\rangle$ on $G$ at $\mathbf{e}$ such that $\psi(U)$ is a transitive rectangular box.

Proof. Let $\langle U, \varphi, n\rangle$ be a chart on $G$ at e. By the previous lemma, there is $g \in U$ such that for $a=\left\langle a_{1}, \ldots, a_{n}\right\rangle=\varphi(g)$ each $a_{i}$ is nontrivial. Let $I_{i}, i=1, \ldots, n$, be open transitive intervals such that $I_{1} \times \cdots \times I_{n} \subseteq \varphi(U)$ and $a \in I_{1} \times \cdots \times I_{n}$. Let $V=a^{-1} \cdot \varphi^{-1}\left(I_{1} \times \cdots \times I_{n}\right)$, and let $\psi: V \rightarrow I_{1} \times \cdots \times I_{n}$ be defined as $x \mapsto \varphi(a x)$. It is easy to see that $\mathfrak{c}=\langle V, \psi \uparrow V, n\rangle$ is a desirable chart.

\section{Groups Definable in O-Minimal EXPANSIONS OF REAL ClOSED FIELDS}

In this section $\mathcal{M}$ will be an o-minimal expansion of a real closed field $\mathcal{R}$.

Let $G$ be a group definable in $\mathcal{M}$. In the case when $\mathcal{R}$ is the field of reals, by Fact 1.10 and Lemma 1.11 every definable group is a Lie group, and every definable homomorphism of two definable groups is Lie. The converse, however, is 
not true. Even in the case when $\mathcal{M}=\mathbb{R}$ there are Lie isomorphic definable groups which are not definably isomorphic. The purpose of this section is to show that a part of classical Lie theory still can be generalized to groups definable in o-minimal expansions of arbitrary real closed fields, when one replaces Lie groups by definable groups and Lie homomorphisms by definable homomorphisms.

Since $\mathcal{M}$ is an o-minimal expansion of a field, it has elimination of imaginaries. It implies, in particular, that whenever $G$ and $H$ are definable groups then we can consider $G / H$ as a definable object.

2.1. Basics of Lie algebras. In this section we will recall some basic properties of Lie algebras. All the facts from this section are well-known. (See [3], for example.)

We fix a field $k=\langle k,+, \cdot\rangle$ of characteristic 0 .

Definition 2.1. A vector space $L$ over $k$ with a bilinear operation [, ] $: L \times L \rightarrow L$ is a Lie algebra over $k$ if $[x, x]=0$ for all $x \in L$ and [, ] satisfies the Jacobi identity:

$$
[x,[y, z]]+[y,[z, x]]+[z,[x, y]]=0 .
$$

We will consider only finite dimensional Lie algebras.

Remark 2.2. If $\langle L,[]$,$\rangle is a Lie algebra over k$ where $L$ is a subspace of $k^{n}$, then $\langle L,[]$,$\rangle is definable in k$. Indeed, if $v_{1}, \ldots, v_{k}$ is a basis for $L$, then, since [, ] is linear, using vectors $v_{i}$ and vectors $u_{i, j}=\left[v_{i}, v_{j}\right]$, it is easy to write a first-order formula that defines $[$,$] .$

Definition 2.3. $\quad$ 1. A subspace $I$ of $L$ is called an ideal of $L$ if $[x, y] \in I$ for all $x \in I, y \in L$.

2. An ideal $I$ of $L$ is abelian if $[x, y]=0$ for all $x, y \in I$.

3. A Lie algebra $L$ is simple if it has no ideals except itself and $\{0\}$.

4. A Lie algebra is semisimple if it has no abelian ideals except for $\{0\}$.

5. A Lie algebra $L$ is the direct sum of its ideals $I_{1}, \ldots, I_{n}$ if $L$ is the direct sum of subspaces $I_{1}, \ldots, I_{n}$.

Fact 2.4. Let $L$ be a semisimple Lie algebra, and let $I$ be an ideal of $L$. Then there is an ideal $J$ of $L$ such that $L$ is the direct sum of $I$ and $J$.

Fact 2.5. Let $L$ be a semisimple Lie algebra. Then there are ideals $L_{1}, \ldots, L_{n}$ of $L$ which are simple, as Lie algebras, such that $L$ is the direct sum of $L_{1}, \ldots, L_{n}$.

Remark 2.6. If $L=\langle V,[]$,$\rangle is a Lie algebra, where V$ is a subspace of $k^{n}$, then $L$ is definable in the field $k$, and the group $\operatorname{Aut}(L)$ of all automorphisms of $L$ is an algebraic subgroup of $\mathrm{GL}(n, k)$.

Fact 2.7. If $L=\langle V,[]$,$\rangle is a semisimple Lie algebra over \mathbb{R}$, where $V$ is a subspace of $\mathbb{R}^{n}$, then $\operatorname{dim}(L)=\operatorname{dim}(\operatorname{Aut}(L))$.

It will be shown later that every definable centerless group can be definably embedded into the group of automorphisms of its Lie algebra. The following claim and some simple dimension arguments, shows that in the case when the Lie algebra is semisimple the range of the embedding is semialgebraic.

Claim 2.8. If $L=\langle V,[]$,$\rangle is a semisimple Lie algebra over a real closed field \mathcal{R}$, where $V$ is a subspace of $\mathcal{R}^{n}$, then $\operatorname{dim}(L)=\operatorname{dim}(\operatorname{Aut}(L))$. 
Proof. Fix $n \in \mathbb{N}$. By the definability of dimension the following statement can be expressed by a first order sentence: If $L=\langle V,[]$,$\rangle is a semisimple Lie algebra over$ a $\mathcal{R}$, where $V$ is a subspace of $\mathcal{R}^{n}$, then $\operatorname{dim}(L)=\operatorname{dim}(\operatorname{Aut}(L))$. Since the first order theories of $\mathcal{R}$ and $\mathbb{R}$ are the same, and this statement holds in $\mathbb{R}$, it is true in $\mathcal{R}$.

2.2. Definable $C^{p}$-actions. In this section we will show that every definable transitive action of a definable group is $C^{p}$ in an appropriate manifold structure.

An action $\alpha: G \times A \rightarrow A$ of a definable group $G$ on a definable set $A$ is $a$ definable action if $\alpha$ is a definable mapping.

Recall that the action $\alpha: G \times A \rightarrow A$ of a group $G$ on a set $A$ is transitive if for all $a, b \in A$ there is $g \in G$ such that $\alpha(g, a)=b$.

The following technical claim will be used in the proof of Theorem 2.11

Claim 2.9. Let $\alpha$ be a definable transitive action of a definable group $G$ on a definable set $A$, and let $C$ be a set such that $G, A$, and $\alpha$ are defined over $C$. If $a \in A$ and $g$ is generic in $G$ over $C \cup\{a\}$, then $\alpha(g, a)$ is generic in $A$ over $C \cup\{a\}$.

Proof. We will assume $C=\varnothing$. Let $K<G$ be the stabilizer of $a$ in $G, n=\operatorname{dim}(G)$, and $k=\operatorname{dim}(K)$. Obviously, $A$ is definably bijective to $G / H$ and, by the dimension formula for quotients (see [5]), $\operatorname{dim}(A)=n-k$. Also, for all $b, c \in A$ the dimension of the set $\{x \in G \mid \alpha(x, b)=c\}$ is equal to $k$.

Let $a_{1}=\alpha(g, a)$. We have $\operatorname{dim}\left(a_{1} g / a\right)=\operatorname{dim}(g / a)=n, \operatorname{dim}\left(a_{1} / a\right) \leq \operatorname{dim}(A)=$ $n-k$, and $\operatorname{dim}\left(g / a_{1} a\right) \leq k$. Since $\operatorname{dim}\left(a_{1} g / a\right)=\operatorname{dim}\left(a_{1} / a\right)+\operatorname{dim}\left(g / a a_{1}\right)$ we have $\operatorname{dim}\left(a_{1} / a\right)=n-k$, and therefore $a_{1}$ is generic in $A$ over $a$.

Definition 2.10. Let $p \geq 0$ be an integer. If $G$ is a definable group and $\mathbf{A}$ is a definable $C^{p}$-manifold, then an action $\alpha: G \times A \rightarrow A$ is called a definable $C^{p}$-action if $\alpha$ is a definable $C^{p}$-mapping. (We consider $G$ as a definable $C^{p}$-group.)

Theorem 2.11. If $A$ is a definable set and $\alpha$ is a definable transitive action of a definable group $G$ on $A$, then for any integer $p \geq 0$ there is a definable $C^{p}$-manifold structure on $A$ such that $f$ is a definable $C^{p}$-action.

Proof. The proof is similar to the proof of Proposition 2.5 in 11, so we will be brief.

We will assume that $\mathcal{M}$ is sufficiently saturated, and that $G, A$ and $\alpha$ are definable over the empty set.

Claim 2.12. If $X$ is a large subset of $A$, then finitely many translates of $X$ cover $A$.

Proof. Since the proof is almost the same as the proof of Lemma 2.4 in [11, we will omit some details.

Let $\mathcal{M}_{0}<\mathcal{M}$ be a small model over which $X$ is defined. It is sufficient to show that for any $a \in A$ there is $g \in G \cap M_{0}$ such that $a \in \alpha(g, X)$. Let $m \in X \cap M_{0}$. Since the action is transitive, there is $h \in G$ such that $a=\alpha(h, m)$. Let $g$ be generic in $G$ over $M_{0} \cup\{h\}$ such that $\operatorname{ta}\left(g / M_{0} \cup\{h\}\right)$ is finitely satisfiable in $M_{0}$. Let $g_{1}=g^{-1} h$. Then $h=g g_{1}$ and $g_{1}$ is generic in $G$ over $M_{0} \cup\{h\}$. Let $b=\alpha\left(g_{1}, m\right)$, so $a=\alpha(g, b)$. As $g_{1}$ is generic over $M_{0}$ and $m \in M_{0}$, by Claim 2.9, $b$ is generic in $A$, and therefore, since $X$ is large in $A, b \in X$.

Proceeding as in the proof of Proposition 2.5 in [11], we can find definable $C^{p}$ charts $\left\langle V_{i}, \varphi_{i}, n\right\rangle, i=1, \ldots r$, such that $V_{i} \cap V_{j}=\varnothing, i \neq j$, and $\bigcup_{i=1}^{r} V_{i}$ is large 
in $G$. Since $\mathcal{M}$ is an expansion of a real closed field we can choose $\varphi_{i}$ so that $\varphi_{i}\left(V_{i}\right) \cap \varphi_{j}\left(V_{j}\right)=\varnothing, i \neq j$. Let $V=\bigcup_{i=1}^{r} V_{i}$ and $\varphi: V \rightarrow M^{n}$ defined as $\varphi \uparrow V_{i}=\varphi_{i}$. Then $V$ is large in $G$ and is in definable bijection with an open subset of $M^{n}$. Without loss of generality we can assume that $V$ is definable over the empty set, $V$ is an open subset of $M^{n}$, and $\varphi$ is the identity mapping.

In the same way we can find a large subset $A_{0}$ of $A$, which is in definable bijection with an open subset of $M^{k}$, such that $A_{0}$ and the bijection are defined over the empty set. For simplicity we will also assume that $A_{0}$ is an open subset of $M^{k}$. Let $A_{1}=\alpha\left(V, A_{0}\right) \cap A_{0}$. Then $A_{1}$ is large in $A$, and, by Fact 1.1, there is large open $Y \subseteq V \times A_{0}$ such that $\alpha(Y) \subseteq A_{1}$ and $\alpha$ is $C^{p}$ on $Y$.

Now we can find a large open subset $X$ of $A_{0}$ such that for every $a \in X$ if $g$ is generic in $G$ over $a$, then $(g, a) \in Y$ and $\left(g, \alpha\left(g^{-1}, a\right)\right) \in Y$.

Claim 2.13. For every $g \in G$ the set $Z=\{x \in X \alpha(g, x) \in X\}$ is open in $X$, and the function $x \mapsto \alpha(g, x)$ is $C^{p}$ on $Z$.

Proof. It is sufficient to show that for every $a \in Z$ the mapping $f: x \mapsto \alpha(g, x)$ is $C^{p}$ at $a$.

Let $a \in Z$ and $b=\alpha(g, a)$. Let $g_{1}, g_{2}$ be generic in $G$ over $\{a, b\}$ such that $g=g_{2} g_{1}$. Since $g_{1}$ is generic over $a\left(g_{1}, a\right) \in Y$ and the function $x \mapsto \alpha\left(g_{1}, x\right)$ is $C^{p}$ at $a$. Let $c=\alpha\left(g_{1}, a\right)$. By the Chain Rule, it is sufficient to show that the function $x \mapsto \alpha\left(g_{2}, x\right)$ is $C^{p}$ at $c$. Since $c=\alpha\left(g_{2}^{-1}, b\right)$ and $g_{2}$ is generic in $G$ over $b$, $\left(g_{2}, c\right) \in Y$.

By Claim 2.12, there are $g_{1}, \ldots, g_{s} \in G$ such that $A=\bigcup_{i=1}^{n} X_{i}$, where $X_{i}=$ $\alpha\left(g_{i}, X\right)$. For $i=1, \ldots, s$ let $\varphi_{i}$ be the function from $X_{i}$ onto $X$ defined as $\varphi_{i}(x)=$ $\alpha\left(g_{i}^{-1}, x\right)$. It follows from Claim 2.13 that the set $\left\{\left\langle X_{i}, \varphi_{i}, k\right\rangle \mid i=1, \ldots, s\right\}$, where $k=\operatorname{dim}(A)$, is a definable $C^{p}$ atlas on $A$, and for any $g \in G$ the function $x \mapsto \alpha(g, x)$ is $C^{p}$ in this manifold structure on $A$. It is routine to check that $\alpha$ is $C^{p}$ action.

Corollary 2.14. If $H$ is a definable subgroup of a definable group $G$, and $p \geq 0$ an integer, then $G / H$ can be equipped with a definable $C^{p}$-manifold structure so that the canonical action of $G$ on $G / H$ is $C^{p}$.

If $\alpha$ is a definable action of a definable group $G$ on a definable set $A$, then for $m \in A$ by $\alpha_{m}$ we will denote the function from $G$ into $A$ defined as $g \mapsto \alpha(g, m)$.

Claim 2.15. Let $\alpha$ be a definable $C^{1}$-action of a definable group $G$ on a definable $C^{1}$-manifold $\mathbf{X}$ and $m \in X$. Then for any $g \in G \operatorname{Rank}\left(\mathrm{d}_{g}\left(\alpha_{m}\right)\right)=\operatorname{Rank}\left(\mathrm{d}_{e}\left(\alpha_{m}\right)\right)$.

Proof. Let $g \in G$. Since

$$
\alpha_{m}(x)=\alpha\left(g g^{-1} x, m\right)=\alpha\left(g, \alpha_{m}\left(g^{-1} x\right)\right)
$$

using the Chain Rule, we obtain

$$
\mathrm{d}_{g}\left(\alpha_{m}(x)\right)=\mathrm{d}_{m}(\alpha(g, x)) \circ \mathrm{d}_{\mathbf{e}}\left(\alpha_{m}(x)\right) \circ \mathrm{d}_{g}\left(g^{-1} x\right) .
$$

Since $\alpha(g, x)$ and $g^{-1} x$ are invertible $C^{1}$-functions whose inverses are $C^{1}$, the linear mappings $\mathrm{d}_{m}(\alpha(g, x))$ and $\mathrm{d}_{g}\left(g^{-1} x\right)$ are invertible, and therefore $\operatorname{Rank}\left(\mathrm{d}_{g}\left(\alpha_{m}\right)\right)=$ $\operatorname{Rank}\left(\mathrm{d}_{\mathbf{e}}\left(\alpha_{m}\right)\right)$.

Corollary 2.16. Let $\alpha$ be a definable $C^{1}$-action of a definable group $G$ on a definable $C^{1}$-manifold $\mathbf{X}, m \in X$, and $H=\{g \in G \mid \alpha(g, m)=m\}$. Then, for any $g \in G$, $\operatorname{Rank}\left(\mathrm{d}_{g}\left(\alpha_{m}\right)\right)=\operatorname{dim}(G)-\operatorname{dim}(H)$, and therefore $\operatorname{dim}\left(\operatorname{ker}\left(\mathrm{d}_{g}\left(\alpha_{m}\right)\right)\right)=\operatorname{dim}(H)$.

Proof. Follows from the above claim and Claim[1.6 


\subsection{Tangent spaces of definable groups.}

Lemma 2.17. If $H$ is a definable subgroup of a definable group $G$, then $H$ is a submanifold of $G$.

Proof. Let $f$ be the identity mapping from $H$ into $G$. We need to show that for any $h \in H$ the rank of $\mathrm{d}_{h}(f)$ is equal to $\operatorname{dim}(H)$.

Let $\alpha: H \times G \rightarrow G$ be the action of $H$ on $G$ by multiplication on the left. It is obvious that $f(x)=\alpha_{\mathbf{e}}(x)$. Since, by Lemma 1.11, $\alpha$ is $C^{1}, \operatorname{Rank}\left(\mathrm{d}_{h}(f)\right)=$ $\operatorname{dim}(H)-0$ for all $h \in H$ by Corollary 2.16

Definition 2.18. For a definable group $G$ the tangent space of $G$ at $\mathbf{e}$ is called the tangent space of $G$.

By the previous lemma, if $H$ is a definable subgroup of a definable group $G$, then the differential of id : $H \rightarrow G$ is an embedding of $\mathcal{T}_{\mathbf{e}}(H)$ into $\mathcal{T}_{\mathbf{e}}(G)$, and we can identify $\mathcal{T}_{\mathbf{e}}(H)$ with a subspace of $\mathcal{T}_{\mathbf{e}}(G)$. Also, for any definable group $G$, since $\mathcal{T}_{\mathbf{e}}(G)$ depends only on the manifold structure of $G$ at $\mathbf{e}$, the tangent space of $G$ is equal to the tangent space of the definably connected component of $G$.

Theorem 2.19. Let $G$ be a definable group, $\alpha$ a definable $C^{1}$-action of $G$ on a definable $C^{1}$-manifold $\mathbf{X}, a \in X$, and $\operatorname{Fix}_{a}(G)=\left\{g \in G \mid \alpha_{a}(g)=a\right\}$.

1. If $H$ is a definably connected definable subgroup of $G$, then $H \subseteq \operatorname{Fix}_{a}(G)$ if and only if $\mathcal{T}_{\mathbf{e}}(H) \subseteq \operatorname{ker}\left(\mathrm{d}_{\mathbf{e}}\left(\alpha_{a}\right)\right)$.

2. The tangent space of $\operatorname{Fix}_{a}(G)$ is $\operatorname{ker}\left(\mathrm{d}_{\mathbf{e}}\left(\alpha_{a}\right)\right)$.

1. If $H \subseteq \operatorname{Fix}_{a}(G)$, then $\alpha_{a}(x)$ is constant on $H$, and therefore $\mathrm{d}_{\mathbf{e}}\left(\alpha_{a}\right)$ is 0 on $\mathcal{T}_{\mathbf{e}}(H)$.

Suppose $\mathcal{T}_{\mathbf{e}}(H) \subseteq \operatorname{ker}\left(\mathrm{d}_{\mathbf{e}}\left(\alpha_{a}\right)\right)$. Let $\beta$ be the restriction of $\alpha$ on $H \times X$. Obviously, $\beta$ is a definable $C^{1}$-action of $H$ on $\mathbf{X}$ with $\operatorname{Rank}\left(\mathrm{d}_{\mathbf{e}}\left(\beta_{a}\right)\right)=0$. Since $H$ is definably connected, by Corollary 2.16 and Claim[1.7, $\beta_{a}(H)=\{a\}$.

2. Applying 1 to the definably connected component of $\operatorname{Fix}_{a}(G)$, we obtain $\mathcal{T}_{\mathbf{e}}\left(\operatorname{Fix}_{a}(G)\right) \subseteq \operatorname{ker}\left(\mathrm{d}_{\mathbf{e}}\left(\alpha_{a}\right)\right)$. By Corollary 2.16

$$
\operatorname{dim}\left(\operatorname{ker}\left(\mathrm{d}_{e}\left(\alpha_{a}\right)\right)\right)=\operatorname{dim}\left(\operatorname{Fix}_{a}(G)\right) .
$$

Since dimension of the group $\operatorname{Fix}_{a}(G)$ is equal to the dimension of its tangent space, the vector spaces $\mathcal{T}_{\mathbf{e}}\left(\operatorname{Fix}_{a}(G)\right)$ and $\operatorname{ker}\left(\mathrm{d}_{\mathbf{e}}\left(\alpha_{a}\right)\right)$ have the same dimension, hence they must be equal.

Claim 2.20. Let $H_{1}$ and $H_{2}$ be definably connected definable subgroups of a definable group $G$. Then $H_{1}=H_{2}$ if and only if $\mathcal{T}_{\mathbf{e}}\left(H_{1}\right)=\mathcal{T}_{\mathbf{e}}\left(H_{2}\right)$.

Proof. Applying Theorem 2.19 to the canonical action of $G$ on the set $G / H_{2}$ of the left cosets of $H_{2}$, we obtain that $H_{1} \subseteq H_{2}$ if and only if $\mathcal{T}_{\mathbf{e}}\left(H_{1}\right) \subseteq \mathcal{T}_{\mathbf{e}}\left(H_{2}\right)$. Similarly, $H_{2} \subseteq H_{1}$ if and only if $\mathcal{T}_{\mathbf{e}}\left(H_{2}\right) \subseteq \mathcal{T}_{\mathbf{e}}\left(H_{1}\right)$.

Theorem 2.21. Let $f: G \rightarrow H$ be a definable homomorphism of definable groups and $H_{1}$ be a definable subgroup of $H$. Then the tangent space of the definable subgroup $G_{1}=f^{-1}\left(H_{1}\right)$ of $G$ is equal to $\left(\mathrm{d}_{\mathbf{e}}(f)\right)^{-1}\left(\mathcal{T}_{\mathbf{e}}\left(H_{1}\right)\right)$.

Proof. Let $\alpha$ be the canonical action of $H$ on $H / H_{1}$. Then $\beta(x, y)=\alpha(f(x), y)$ is a definable $C^{1}$-action of $G$ on $H / H_{1}$ and $G_{1}=\operatorname{Fix}_{H_{1}}(G)$. By Theorem 2.19

$$
\mathcal{T}_{\mathbf{e}}\left(G_{1}\right)=\operatorname{ker}\left(\mathrm{d}_{\mathbf{e}}\left(\beta_{H_{1}}\right)\right)=\operatorname{ker}\left(\mathrm{d}_{\mathbf{e}}\left(\alpha_{H_{1}}\right) \circ \mathrm{d}_{\mathbf{e}}(f)\right) .
$$

Since $\operatorname{ker}\left(\mathrm{d}_{\mathbf{e}}\left(\alpha_{H_{1}}\right)\right)=\mathcal{T}_{\mathbf{e}}\left(H_{1}\right)$, we have $\mathcal{T}_{\mathbf{e}}\left(G_{1}\right)=\left(\mathrm{d}_{\mathbf{e}}(f)\right)^{-1}\left(\mathcal{T}_{\mathbf{e}}\left(H_{1}\right)\right)$. 
Corollary 2.22. If $f$ is a definable automorphism of a definable group $G$, then $\mathrm{d}_{\mathbf{e}}(f)$ is an automorphism of $\mathcal{T}_{\mathbf{e}}(G)$

Proof. $\mathrm{d}_{\mathbf{e}}(f)$ is a linear transformation of a finite dimensional vector space whose kernel is trivial by Theorem 2.21 .

Remark 2.23. 1. Let $\mathrm{L}(n, \mathcal{R})$ be the space of all square matrices of order $n$ over $\mathcal{R}$. We will identify $\mathrm{L}(n, \mathcal{R})$ with $\mathcal{R}^{n^{2}}$ in the obvious way. The group $\operatorname{GL}(n, R)$ of all invertible matrices has a canonical definable $C^{p}$-atlas with a single chart $\mathfrak{c}=\left\langle G L(n, \mathcal{R}), \varphi, n^{2}\right\rangle$, where $\varphi: A \mapsto A-I_{n}$, and the tangent space of $\operatorname{GL}(n, R)$, by means of $\Theta_{\mathfrak{c}}$, can be identified with $\mathrm{L}(n, \mathcal{R})$.

2. Let $V$ be an $n$-dimensional vector space over $\mathcal{R}$. After fixing a basis for $V$, we can identify, in the obvious way, the group $\operatorname{Aut}(V)$ with $\operatorname{GL}(n, \mathcal{R})$, and the group $\operatorname{End}(V)$ with $\mathrm{L}(n, \mathcal{R})$. If $f$ is a function from a definable set $X$ into $\operatorname{Aut}(V)$ (or $\operatorname{End}(V)$ ), then we say that $f$ is definable if, after the above identification, the corresponding function from $X$ into $\mathrm{GL}(n, \mathcal{R})$ (or $\mathrm{L}(n, \mathcal{R})$ ) is definable. In the same manner we transfer the notion of a definable $C^{p}$-function to functions whose domain and range can be $\operatorname{Aut}(V)$ or $\operatorname{End}(V)$. Thus if $f$ is a definable $C^{1}$-function from a definable group $G$ into $\operatorname{Aut}(V)$, then we can consider its differential at $\mathbf{e}$ as a definable linear function from $\mathcal{T}_{\mathbf{e}}(G)$ into $\operatorname{End}(V)$. Moreover, using the change of a basis formula, it is easy to see that all these notions are independent of the choice of basis.

Theorem 2.24. Let $G$ be a definable group, $V$ an $n$-dimensional vector space over $\mathcal{R}, f: G \rightarrow \operatorname{Aut}(V)$ a definable homomorphism, and $U \subset V$ a subspace.

1. The tangent space of the definable subgroup $G_{1}=\{g \in G \mid f(g)(U) \subseteq U\}$ is $\mathcal{T}_{\mathbf{e}}\left(G_{1}\right)=\left\{\xi \in \mathcal{T}_{\mathbf{e}}(G) \mid \mathrm{d}_{\mathbf{e}}(f)(\xi)(U) \subseteq U\right\}$.

2. The tangent space of the definable subgroup $G_{2}=\left\{g \in G|f(g)| U=\mathrm{id}_{U}\right\}$ is $\mathcal{T}_{\mathbf{e}}\left(G_{2}\right)=\left\{\xi \in \mathcal{T}_{\mathbf{e}}(G) \mid \mathrm{d} \mathbf{e}(f)(\xi)\lceil U=0\}\right.$.

Proof. 1. Let $H_{1}=\{A \in \operatorname{Aut}(V) \mid A(U) \subseteq U\}$ and $h_{1}=\{A \in \operatorname{End}(V) \mid A(U) \subseteq U\}$. Obviously, $h_{1}$ is a subspace of $\operatorname{End}(U)$, and $H_{1}$ is an open subset of $h_{1}$. Hence $h_{1}$ is the tangent space of $H_{1}$, and we can apply Theorem 2.21 to $f$ and $H_{1}$.

2. Let $H_{2}=\{A \in \operatorname{Aut}(V) \mid(A-E)(U)=0\}$ and $h_{2}=\{A \in \operatorname{End}(V) \mid A(U)=0\}$. Obviously, $h_{2}$ is a subspace of $\operatorname{End}(U)$, and $H_{2}-E$ is an open subset of $h_{2}$. Hence, $h_{2}$ is the tangent space of $H_{2}$, and we can apply Theorem 2.21 to $f$ and $H_{2}$.

2.4. Lie algebras of definable groups. The following fact follows from Lemma 3.2 in 7 .

Fact 2.25. Let $f_{1}$ and $f_{2}$ be definable endomorphisms of a definable group $G$. Then $f_{1} \uparrow G^{0}=f_{2} \uparrow G^{0}$ if and only if $\mathrm{d}_{\mathbf{e}}\left(f_{1}\right)=\mathrm{d} \mathbf{e}\left(f_{2}\right)$.

Let $G$ be a definable group.

For $g \in G$, by $a(g)$ we will denote the inner automorphism

$$
a(g): x \mapsto g^{-1} x g .
$$

Let $\operatorname{Ad} g=\mathrm{d}_{\mathbf{e}}(a(g))$. By Corollary 2.22, $\operatorname{Ad} g \in\left(\operatorname{Aut}\left(\mathcal{T}_{\mathbf{e}}(G)\right)\right)$ and hence

$$
\text { Ad }: G \rightarrow \operatorname{Aut}\left(\mathcal{T}_{\mathbf{e}}(G)\right)
$$

is a definable homomorphism.

Claim 2.26. $\operatorname{ker}(A d)=\mathbf{C}\left(G^{0}\right)$. 
Proof. Follows from Fact 2.25

Let $\operatorname{ad}=\mathrm{d}_{\mathbf{e}}(\mathrm{Ad})$. We have

$$
\operatorname{ad}: \mathcal{T}_{\mathbf{e}}(G) \rightarrow \operatorname{End}\left(\mathcal{T}_{\mathbf{e}}(G)\right) .
$$

On the tangent space $\mathcal{T}_{\mathbf{e}}(G)$ we define a binary operation $[$,$] as [\xi, \zeta]=\operatorname{ad}(\xi)(\zeta)$.

Claim 2.27. $\left\langle\mathcal{T}_{\mathbf{e}}(G),[],\right\rangle$ is a Lie algebra.

Proof. Let $\mathfrak{c}=\langle U, \varphi, n\rangle$ be a definable $C^{3}$-chart on $G$ at $\mathbf{e}$ with $\varphi(\mathbf{e})=0$. Using Taylor series we obtain

$$
\varphi\left(g_{1} \cdot g_{2}\right)=\varphi\left(g_{1}\right)+\varphi\left(g_{2}\right)+\alpha\left(\varphi\left(g_{1}\right), \varphi\left(g_{2}\right)\right)+\ldots
$$

where $\alpha$ is a bilinear form and ... stands for elements of order greater than 2. Using elementary calculus (see [6] for details), it can be shown that $[x, y]=\alpha(x, y)-$ $\alpha(y, x)$.

Definition 2.28. For a definable group $G$ the Lie algebra $\left\langle\mathcal{T}_{\mathbf{e}}(G),[],\right\rangle$ is called the Lie algebra of $G$.

Claim 2.29. Let $G$ be a definable group and $\mathfrak{g}$ its Lie algebra.

1. If $f$ is a definable automorphism of $G$, then $\mathrm{d}_{\mathbf{e}}(f)$ is a definable automorphism of $\mathfrak{g}$.

2. $\operatorname{Ad}(g)$ is an automorphism of $\mathfrak{g}$ for every $g \in G$.

Proof. 1 can be shown using basic calculus; see [6] for details.

2 follows from 1.

Claim 2.30. If $H$ is a definable subgroup of a definable group $G$, then the Lie algebra of $H$ is a Lie subalgebra of the Lie algebra of $G$.

Proof. Obvious.

Applying Theorem 2.24 to the adjoint representation we obtain

Claim 2.31. Let $\mathfrak{g}$ be the Lie algebra of a definable group $G$ and $\mathfrak{h}$ be a subspace of $\mathfrak{g}$. Then

1. the subalgebra $\{\xi \in \mathfrak{g} \mid[\xi, \mathfrak{h}]=0\}$ is the Lie algebra of the subgroup $\{g \in G \mid \operatorname{Ad} g\lceil\mathfrak{h}=\mathrm{id}\}$.

2. the subalgebra $\{\xi \in \mathfrak{g} \mid[\xi, \mathfrak{h}] \subseteq \mathfrak{h}\}$ is the Lie algebra of the subgroup $\{g \in G \mid \operatorname{Ad} g(\mathfrak{h}) \subseteq \mathfrak{h}\}$.

Claim 2.32. Let $G$ be a definably connected definable group with Lie algebra $\mathfrak{g}$.

1. $G$ is abelian if and only if $\mathfrak{g}$ is an abelian Lie algebra.

2. If $H$ is a definably connected definable subgroup of $G$, then $H$ is a normal subgroup of $G$ if and only if its Lie algebra $\mathfrak{h}$ is an ideal of $\mathfrak{g}$.

Proof. 1. Easy.

2. By Claim 2.20, $H$ is normal if and only if $\operatorname{Ad}(g)(\mathfrak{h}) \subseteq \mathfrak{h}$ for any $g \in G$. Now apply Claim 2.31. 
2.5. Definable semisimple groups. Recall that if $G$ is a connected Lie group over $\mathbb{R}$, then $G$ is called semisimple if all its abelian normal subgroups are discrete. By Corollary 1.17, if a group $G$ is definable in an o-minimal expansion of $\mathbb{R}$ and is definably connected, then $G$ is semisimple if and only if it does not have an infinite abelian normal subgroup. Thus the following definition is a generalization of semisimplicity to groups definable in o-minimal structures.

Definition 2.33. A definably connected group $G$ definable in an o-minimal structure is called semisimple if it has no infinite abelian normal subgroup.

Theorem 2.34. Let $G$ be a definably connected definable group. $G$ is semisimple if and only if its Lie algebra $\mathfrak{g}$ is semisimple.

Proof. If $\mathfrak{g}$ is semisimple, then, by Claim 2.32 $G$ is semisimple.

Now we assume that $\mathfrak{g}$ is not semisimple and will show that $G$ is not semisimple. Let $\mathfrak{j} \subseteq \mathfrak{g}$ be a nontrivial abelian ideal. Let $H=\{g \in G \mid \operatorname{Ad}(g) \uparrow \mathfrak{j}=\mathrm{id}\}$. By Claim [2.31] the tangent algebra of $H$ is $\mathfrak{h}=\{\xi \in \mathfrak{g} \mid[\xi, \mathfrak{j}]=0\}$. Since $\mathfrak{j}$ is an abelian ideal, using the Jacobi identity, it is not hard to see that $\mathfrak{h}$ is an ideal of $\mathfrak{g}$ containing $\mathfrak{j}$. By Claim 2.32 the definable connected component $H^{0}$ of $H$ is a definable normal subgroup of $G$. Let $C=\left\{h \in H^{0} \mid A d(h)\lceil\mathfrak{h}=\right.$ id $\}$. It is easy to check that its Lie algebra $\mathfrak{c}=\{\xi \in \mathfrak{h} \mid[\xi, \mathfrak{h}]=0\}$ is an abelian ideal of $\mathfrak{g}$. Hence the definably connected component $C^{0}$ of $C$ is a normal abelian subgroup of $G$. Since the Lie algebra of $C^{0}$ contains $\mathfrak{j}, C^{0}$ is nontrivial.

Claim 2.35. Let $G$ be a definably connected definable semisimple group with Lie algebra $\mathfrak{g}$. If $\mathfrak{h}$ is an ideal of $\mathfrak{g}$, then there is a definably connected definable normal subgroup $H$ of $G$ whose Lie algebra is $\mathfrak{h}$.

Proof. Since $G$ is semisimple its Lie algebra is semisimple, and, by Fact 2.4 , there is an ideal $\mathfrak{i}$ of $\mathfrak{g}$ such that $\mathfrak{g}$ is the direct sum of $\mathfrak{h}$ and $\mathfrak{i}$. Using semisimplicity of $\mathfrak{g}$, it is easy to see that $\mathfrak{h}=\{h \in \mathfrak{g} \mid[h, \mathfrak{i}]=0\}$. By Claim 2.31 $\mathfrak{h}$ is the Lie algebra of the definable subgroup $H_{1}=\{h \in G \mid \operatorname{Ad} h\lceil\mathfrak{h}=\mathrm{id}\}$. By Claim 2.32, the definably connected component $H$ of $H_{1}$ is a normal subgroup of $G$.

Theorem 2.36. Let $G$ be a definably connected centerless definable group. $G$ is definably simple if and only if its Lie algebra $\mathfrak{g}$ is simple.

Proof. If $G$ is definably simple, then it is semisimple, and, by Claim 2.35, its Lie algebra is simple.

Assume $\mathfrak{g}$ is simple and let us show that $G$ is definably simple. Suppose $H$ is a proper normal definable subgroup of $G$. Since $G$ is definably connected, $H$ has an infinite index in $G$, and Lie algebra $\mathfrak{h}$ of $H$ is a proper ideal of $\mathfrak{g}$. Since $\mathfrak{g}$ is simple $\mathfrak{h}=0$, hence $H$ is finite. For any $h \in H$ the function $f: g \mapsto g^{-1} h g$ is a definable continuous function from $G$ into $H$. As $G$ is definably connected and $H$ is finite, $f$ must be constant, and therefore $G$ commutes with every element in $H$. Since $G$ is centerless $H$ must be trivial.

Theorem 2.37. Let $G$ be a centerless definable group such that $G^{0}$ has no nontrivial abelian normal subgroup. Then there is a linear semialgebraic group $H$ over $\mathcal{R}$ definably isomorphic to $G$.

Proof. Let $\mathfrak{g}=\left\langle\mathcal{T}_{\mathbf{e}}(G),[],\right\rangle$ be the Lie algebra of $G$. Fixing a basis of $\mathcal{T}_{\mathbf{e}}(G)$, we may assume that $\mathcal{T}_{\mathbf{e}}(G)=\mathcal{R}^{n}$ and Ad is a definable homomorphism from $G$ 
into $\operatorname{GL}(n, \mathcal{R})$. Let $H=\operatorname{Ad}(G)$. Since $G$ is centerless, By Claim 2.26] Ad is an isomorphism from $G$ onto $H$, and it is sufficient to show that $H$ is a semialgebraic subset of $\operatorname{GL}(n, \mathcal{R})$.

Let $A=\operatorname{Aut}(\mathfrak{g})<\operatorname{GL}(n, \mathcal{R})$. By Claim [2.29 $H$ is a subgroup of $A$. Since $G^{0}$ is semisimple, by Theorem 2.34 and Claim 2.8 .

$$
\operatorname{dim}(\operatorname{Aut}(\mathfrak{g}))=\operatorname{dim}(\mathfrak{g})=\operatorname{dim}(G)=\operatorname{dim}(H),
$$

hence $H$ has a finite index in $A$. Let $A^{0}$ be the definably connected component of A. By Claim 1.3, $A^{0}$ is semialgebraic. Since $A^{0}<H$ and $\operatorname{dim}\left(A^{0}\right)=\operatorname{dim}(H), H$ consists of finitely many classes of $A^{0}$, and therefore it is semialgebraic.

Theorem 2.38. If $G$ is a definably connected centerless and semisimple definable group, then $G$ is the direct product of definably simple definable subgroups.

Proof. Let $\mathfrak{g}$ be the Lie algebra of $G$. By Fact [2.5 $\mathfrak{g}$ is the direct sum of ideals $\mathfrak{h}_{1}, \ldots, \mathfrak{h}_{k}$ such that each $\mathfrak{h}_{i}$ is a simple Lie algebra.

By Claim 2.35, there are normal definably connected definable subgroups $H_{1}, \ldots, H_{k}$ such that $\mathfrak{h}_{i}$ is the Lie algebra of $H_{i}$ for every $i \in\{1, \ldots, k\}$. Hence, by Theorem 2.36 each $H_{i}$ is definably simple. We want to show that $G$ is the direct product of $H_{1}, \ldots, H_{k}$.

Let $i \neq j \in\{1, \ldots, k\}$. Since $\left[\mathfrak{h}_{i}, \mathfrak{h}_{j}\right]=0$, by Claim 2.31

$$
H_{i} \subseteq\left\{g \in G \mid \operatorname{Ad}(g)\left\lceil\mathfrak{h}_{j}=\mathrm{id}\right\}\right.
$$

and hence, by Fact 2.25, every $h \in H_{i}$ commutes with $H_{j}$.

Since each $H_{i}$ is definably simple $H_{i} \cap H_{j}=\{\mathbf{e}\}$ for $i \neq j$. Thus $H=H_{1} \cdot H_{2} \cdot \ldots$. $H_{k}$ is the direct product of $H_{1}, \ldots, H_{k}$ and $\operatorname{dim}(H)=\operatorname{dim}\left(H_{1}\right)+\ldots+\operatorname{dim}\left(H_{k}\right)$. On the other hand, $\operatorname{dim}(G)=\operatorname{dim}(\mathfrak{g})=\operatorname{dim}\left(\mathfrak{h}_{1}\right)+\ldots+\operatorname{dim}\left(\mathfrak{h}_{k}\right)$. Therefore $\operatorname{dim}(H)=$ $\operatorname{dim}(G)$ and, since $G$ is definably connected, $G=H$.

\section{A DECOMPOSITION OF CENTERLESS GROUPS}

In this section we will prove the following two theorems.

Theorem 3.1. If $G$ is a definable $\mathbb{G}$-definably connected centerless group, then $G$ is the direct product of $\mathbb{G}$-definable unidimensional subgroups.

Theorem 3.2. If $G$ is a definable $\mathbb{G}$-definably connected unidimensional centerless group, then there is a definable real closed field $\mathcal{R}$ and a definable linear group $H<\mathrm{GL}(n, \mathcal{R})$ definably isomorphic to $G$.

Before proving the above theorems we establish some conventions.

In this section $G$ will be a fixed $\mathbb{G}$-definably connected centerless group definable in $\mathcal{M}$, and $n$ will be the dimension of $G$. We use $\star$ to denote the group multiplication of $G$, and $\mathbf{e}$ will be the identity element of $G$.

By Lemma 1.28, there is a definable chart $\langle B, \varphi, n\rangle$ on $G$ at $\mathbf{e}$ such that $\varphi(B)$ is a transitive rectangular box. We fix one such chart, and, for simplicity, we will assume that $B$ is an open subset of $M^{n}$ and $\varphi$ is the identity mapping. Let $I_{1}, \ldots, I_{n}$ be open transitive intervals such that $B=I_{1} \times \ldots I_{n}$.

We also fix an open rectangular box $U \subseteq B$ containing e such that $a \star b \star c^{-1} \in B$ and $a^{-1} \star b \in B$ for all $a, b, c \in U$.

We will assume that $G$ and the sets $B, U$ are definable over the empty set. 
3.1. Proof of Theorem 3.1. By an easy induction on $n$, Theorem 3.1 follows from the next lemma.

Lemma 3.3. Let $\{1, \ldots, n\}=s^{1} \cup s^{2}$ be a partition of $\{1, \ldots, n\}$ into two nonempty disjoint subsets. If $D_{1}=\Pi_{i \in s^{1}} I_{i}$ is orthogonal to $D_{2}=\Pi_{i \in s^{2}} I_{i}$, then there are $\mathbb{G}$ definable subgroups $H_{1}, H_{2}<G$ such that $G$ is the direct product of $H_{1}$ and $H_{2}$, $\operatorname{dim}\left(H_{1}\right)=\left|s^{1}\right|$ and $\operatorname{dim}\left(H_{2}\right)=\left|s^{2}\right|$.

Proof. Let $e_{i} \in I_{i}, i=1, \ldots, n$ be such that $\mathbf{e}=\left\langle e_{1}, \ldots, e_{n}\right\rangle$. For $k \in\{1,2\}$ we define a function $\rho_{k}: B \rightarrow B$ in the following way: if $a=\left\langle a_{1}, \ldots, a_{n}\right\rangle, b=$ $\left\langle b_{1}, \ldots, b_{n}\right\rangle \in B$, then

$$
\rho_{k}(a)=b \text { iff } b_{i}= \begin{cases}a_{i} & \text { if } i \in s^{k} \\ e_{i} & \text { otherwise. }\end{cases}
$$

Obviously, $\rho_{k} \circ \rho_{k}=\rho_{k}$ and $\rho_{1}(B) \cap \rho_{2}(B)=\{\mathbf{e}\}$.

Let $B_{k}=\rho_{k}(B)$ and $U_{k}=\rho_{k}(U)$. We have $U_{k} \subseteq B_{k}, \rho_{k} \uparrow B_{k}=\operatorname{id}_{B_{k}}$ and $B_{1} \cap B_{2}=\{\mathbf{e}\}$.

Claim 3.4. Let $k \in\{1,2\}$ and $b \in B$.

1. $b \in B_{k}$ if and only if $\rho_{k}(b)=b$.

2. $\rho_{1}(b)=\mathbf{e}$ if and only if $b \in B_{2}$; and $\rho_{2}(b)=\mathbf{e}$ if and only if $b \in B_{1}$.

3. The projection of $B$ onto $s^{k}$-coordinates maps $B_{k}$ homeomorphicly onto $D_{k}$ and $U_{k}$ onto an open rectangular box.

4. If $f$ is a definable continuous function from $U_{1}$ into $B_{2}$, then $f$ is a constant function; and the same holds for definable continuous functions from $\mathrm{U}_{2}$ into $B_{1}$.

Proof. 1-3 are easy. 4 follows from 3, Claim 1.19 and Claim 1.20 ,

Claim 3.5. Let $k \in\{1,2\}$ and let $f: U_{k} \rightarrow B$ be a definable continuous function. If $f(a) \in B_{k}$ for some $a \in U_{k}$, then $f\left(U_{k}\right) \subseteq B_{k}$.

Proof. Assume $k=1$. Since $f$ is continuous, by Claim [3.4(4), the function $\rho_{2} \circ f$ must be constant on $U_{1}$, and, since $\rho_{2}(f(a))=\mathbf{e}$, it must be equal to $\mathbf{e}$ on $U_{1}$. By Claim [3.4 2$), f(b) \in B_{1}$ for all $b \in U_{1}$.

Claim 3.6. Let $k \in\{1,2\}$. If $a, b \in U_{k}$, then $a \star b^{-1} \in B_{k}$ and $a^{-1} \star b \in B_{k}$.

Proof. We assume $k=1$ and will only show that $a \star b^{-1} \in B_{k}$.

Consider the function $f: x \mapsto a \star x^{-1}$. Since $\mathbf{e} \in U_{1}$ and $f(\mathbf{e})=a \in B_{1}$, by Claim 3.5 $f\left(U_{1}\right) \subseteq B_{1}$.

Claim 3.7. If $a \in U_{1}$ and $b \in U_{2}$, then $a \star b=b \star a$.

Proof. Let $d \in U_{2}$. Consider the function $f: x \mapsto d \star x \star d^{-1}$. Since $f(\mathbf{e})=\mathbf{e} \in B_{1}$, by Claim 3.5 $d \star c \star d^{-1} \in B_{1}$ for all $c \in U_{1}$. As $d$ was arbitrary, we have

$$
=\forall x \in U_{2} \forall y \in U_{1} x \star y \star x^{-1} \in B_{1} .
$$

Hence $g: x \mapsto x \star a \star x^{-1}$ is a continuous function from $U_{2}$ into $B_{1}$ that must be constant. So $g(b)=g(\mathbf{e})=a$ and $a \star b=b \star a$.

Claim 3.8. Let $a, c \in U_{1}$ and $b, d \in U_{2}$. If $a \star b=c \star d$, then $a=c$ and $b=d$.

Proof. We have $c^{-1} \star a=d \star b^{-1}$. By Claim 3.6, $c^{-1} \star a \in B_{1}$ and $d \star b^{-1} \in B_{2}$. Since $B_{1} \cap B_{2}=\{\mathbf{e}\}$, we have $c^{-1} \star a=d \star b^{-1}=\mathbf{e}$. 
Claim 3.9. $\operatorname{dim}\left(U_{1} \star U_{2}\right)=n$.

Proof. Since $n$ is the maximal possible dimension for definable subsets of $G$, we only need to show that $\operatorname{dim}\left(U_{1} \star U_{2}\right) \geq n$.

Since $\operatorname{dim}\left(U_{1}\right)=\left|s^{1}\right|$ and $\operatorname{dim}\left(U_{2}\right)=\left|s^{2}\right|=n-\left|s^{1}\right|$, dimension of the Cartesian product of $U_{1}$ and $U_{2}$ is $n$. By the previous claim, $\langle x, y\rangle \mapsto x \star y$ is a definable injective map from $U_{1} \times U_{2}$ into $U_{1} \star U_{2}$. Therefore $\operatorname{dim}\left(U_{1} \star U_{2}\right) \geq n$.

Now we are ready to finish the proof of Lemma 3.3

Let $H_{1}=\mathbf{C}_{G}\left(U_{2}\right)$ and $H_{2}=\mathbf{C}_{G}\left(H_{1}\right)$. By Corollary 1.16, $H_{1}$ and $H_{2}$ are $\mathbb{G}$ definable subgroups. Obviously, $H_{1}$ and $H_{2}$ commute, $U_{2} \subseteq H_{2}$, and, by Claim 3.7 $U_{1} \subseteq H_{1}$. Thus $\operatorname{dim}\left(H_{1}\right) \geq \operatorname{dim}\left(U_{1}\right)=\left|s^{1}\right|$ and $\operatorname{dim}\left(H_{2}\right) \geq \operatorname{dim}\left(U_{2}\right)=\left|s^{2}\right|$.

If $a \in H_{1} \cap H_{2}$, then $a \in \mathbf{C}_{G}\left(U_{2}\right) \cap \mathbf{C}_{G}\left(U_{1}\right)$. Hence $U_{1} \star U_{2} \subseteq \mathbf{C}_{G}(a)$ and, since $G$ is $\mathbb{G}$-definably connected, by Claim 3.9 and Fact $1.14 \mathbf{C}_{G}(a)=G$. As $G$ is centerless, $a=\mathbf{e}$. Thus $H_{1} \cap H_{2}=\{\mathbf{e}\}, H_{1} \star H_{2}$ is the direct product of $H_{1}$ and $H_{2}$, and $\operatorname{dim}\left(H_{1} \star H_{2}\right)=\operatorname{dim}\left(H_{1}\right)+\operatorname{dim}\left(H_{2}\right)$.

Since $\operatorname{dim}\left(H_{1} \star H_{2}\right) \leq n, \operatorname{dim}\left(H_{1}\right) \geq\left|s^{1}\right|, \operatorname{dim}\left(H_{2}\right) \geq\left|s^{2}\right|$, and $\left|s^{1}\right|+\left|s^{2}\right|=n$, we obtain $\operatorname{dim}\left(H_{1}\right)=\left|s^{1}\right|, \operatorname{dim}\left(H_{2}\right)=\left|s^{2}\right|$ and $\operatorname{dim}\left(H_{1} \star H_{2}\right)=n$. Hence $H_{1} \star H_{2}$ has finite index in $G$, and therefore $G=H_{1} \star H_{2}$.

3.2. Proof of Theorem 3.2. Assume $G$ is $\mathbb{G}$-definably connected, centerless and unidimensional.

Since it is sufficient to prove the theorem for an $\omega_{1}$-saturated elementary extension of $\mathcal{M}$, we will assume $\mathcal{M}$ is $\omega_{1}$-saturated.

By Claim 1.26 2), we can assume $B=I^{n}$, where $I$ is an open transitive interval, and $\mathbf{e}=\langle d, \ldots, d\rangle$ for some $d \in I$.

We will prove Theorem 3.2 by first showing that there is a real closed field $\mathcal{R}$ definable on a subinterval of $I$, and then, using the adjoint mapping, we will embed $G$ into $\operatorname{GL}(n, \mathcal{R})$.

3.2.1. Finding a field. Since $d$ is a nontrivial element, by the Trichotomy Theorem [10], exactly one of the following two cases holds:

Case 1: There is a definable real closed field on a subinterval of $I$ containing $d$.

Case 2: The structure that $\mathcal{M}$ induces on some open convex neighborhood of $d$ is an ordered vector space over an ordered division ring.

Let $\mathcal{V}$ be an ordered vector space over an ordered division ring $D$. By Propositions 3.9, 3.10 in [5], if $f: \mathcal{V} \rightarrow \mathcal{V}$ is a function definable in $\mathcal{V}$ with $f(0)=0$, then there is $\lambda \in D$ such that $f(x)=\lambda(x)$ for all $x>0$ close enough to 0 .

Thus, in case 2 , if $f$ is a definable continuous function from $I$ into $M^{n}$ with $f(d)=\langle d, \ldots, d\rangle$, then there is a function $\alpha$ definable over $\{d\}$ such that $f(x)=$ $\alpha(x)$ for all $x>d$ close enough to $d$.

Hence, by the Compactness Theorem, to rule out Case 2 it is sufficient to find a definable function $F(x, y)$ and an infinite set $C$ (not necessarily definable), such that the following three conditions hold:

(a) $F(x, c)$ is a continuous function from $I$ into $M^{n}$ for every $c \in C$;

(b) $F(d, c)=\langle d, \ldots, d\rangle$ for every $c \in C$;

(c) If $c_{1} \neq c_{2} \in C$, then $F\left(x, c_{1}\right) \neq F\left(x, c_{2}\right)$ for all $x>d$ close enough to $d$.

Let $I^{+}=\{b \in I \mid b>d\}$ and for $b \in I^{+}$we will denote the interval $(d, b)$ by $I_{b}$.

Notice that, by o-minimality, the condition (c) above is equivalent to 
(c') If $c_{1} \neq c_{2} \in C$, then for any $b \in I^{+}$there is $x \in I_{b}$ such that $F\left(x, c_{1}\right) \neq$ $F\left(x, c_{2}\right)$.

Let $\rho: I \rightarrow B$ be the continuous injection defined as $\rho(x)=\langle x, d, \ldots, d\rangle$. For $b \in I^{+}$let $\hat{I}_{b}$ denote the set $\rho\left(I_{b}\right)$, and let $H_{b}$ be the centralizer of $\hat{I}_{b}$ in $G$. It is easy to see that if $b^{1}<b^{2}$, then $H_{b^{2}} \subseteq H_{b^{1}}$.

Let $H=\bigcup_{b \in I^{+}} H_{b}$.

Claim 3.10. There is $p \in I^{+}$such that $H=H_{p}$.

Proof. Since $H_{b} \subseteq H$ for every $b \in I^{+}$, it is sufficient to find $p \in I^{+}$such that $H \subseteq H_{p}$.

Obviously, $\mathbf{C}_{G}(H)=\bigcap_{b \in I^{+}} \mathbf{C}_{G}\left(H_{b}\right)$. Hence, by Fact 1.13, there is $p \in I^{+}$ such that $\mathbf{C}_{G}(H)=\mathbf{C}_{G}\left(H_{p}\right)$. Since $\hat{I}_{p} \subseteq \mathbf{C}_{G}\left(H_{p}\right)$, we have $\hat{I}_{p} \subseteq \mathbf{C}_{G}(H)$ and $H \subseteq \mathbf{C}_{G}\left(\hat{I}_{p}\right)=H_{p}$.

Remark 3.11. The above proof actually shows that if a group $G$ satisfies the DCC condition on $\mathbb{G}$-definable subgroups, then $G$ satisfies the ACC condition on centralizers.

By Claim $3.10, H$ is $\mathbb{G}$-definable.

Since $G$ is centerless and definably connected, $\operatorname{dim}(H)<\operatorname{dim}(G)$. (Otherwise $H$ would be equal to $G$, and $\hat{I}_{p}$ would be in the center of $G$.) Hence $I^{n}$ cannot be covered by finitely many left cosets of $H$, and we can find infinite set $C \subseteq I^{n}$ such that $c_{1} \star H \neq c_{2} \star H$ for $c_{1} \neq c_{2} \in C$.

It is easy to check that $C$ and the function $F(x, y)=y \star \rho(x) \star y^{-1}$ satisfy the conditions (a), (b), (c') above.

Thus case 2 is impossible, and there must be a real closed field $\mathcal{R}$ definable on a subinterval of $I$ containing $d$.

3.2.2. Embedding $G$ into $\mathrm{GL}(n, \mathcal{R})$. Decreasing the interval $I$, if needed, we assume that a real closed field $\mathcal{R}$ is definable on $I$.

If $\mathfrak{c}=\langle V, \varphi, n\rangle$ is a definable chart on $G$ with $\varphi(V) \subseteq I^{n}$, and $f\left(x_{1}, \ldots, x_{k}\right)$ is a function from an open $D \subseteq V^{k}$ into $V$, then we say that $f$ is a $C^{1}$-function relative to $\mathfrak{c}$ if $\varphi\left(f\left(\varphi^{-1}\left(x_{1}\right), \ldots, \varphi^{-1}\left(x_{k}\right)\right)\right)$ is a $C^{1}$-function (in the sense of $\mathcal{R}$ ) from $\varphi(D)$ into $\varphi(V)$.

Claim 3.12. There is a definable chart $\mathfrak{c}=\langle V, \varphi, n\rangle$ on $G$ at $\mathbf{e}$ and an open set $V_{0} \subseteq V$ containing e such that $\varphi(V) \subseteq I^{n}$ and, relative to $\mathfrak{c}$, group multiplication and inversion are $C^{1}$-functions from $V^{0} \times V^{0}$ and $V^{0}$, respectively, into $V$.

Proof. By the implicit function theorem, it is sufficient to prove only that there is a chart $\mathfrak{c}$, as in the statement of the claim, such that the group multiplication is $C^{1}$.

For $a \in I^{n}$ let $\varphi_{a}(x)=a \star x$. Since the group operations are continuous, for every $a \in I^{n}$, there is an open set $D_{a} \subseteq I^{n}$ containing e such that $\varphi_{a}\left(D_{a}\right) \subseteq I^{n}$, and therefore $\left\langle D_{a}, \varphi_{a}, n\right\rangle$ is a definable chart on $G$ at $\mathbf{e}$ with $\varphi_{a}\left(D_{a}\right) \subseteq I^{n}$.

Thus it suffices to show that there is $a \in I^{n}$ and an open set $U \subseteq I^{n}$ containing $a$ such that the function $(x, y) \mapsto x \star a^{-1} \star y$ is $C^{1}$ on $U \times U$.

Let $b, c$ be independent generic elements in $I^{n}$ such that $c \star b \in I^{n}$. Let $a=c \star b$, $h(x, y)=x \star a^{-1} \star y, f_{1}(x)=x \star b^{-1}, f_{2}(x)=c^{-1} \star x$, and $F(x, y)=x \star y$. Then $h(x, y)=F\left(f_{1}(x), f_{2}(y)\right)$ and, by the Chain Rule, it is sufficient to show that there 
is an open set $U_{1}$ containing $c$, an open set $U_{2}$ containing $b$, and an open set $U$ containing $a$, such that $F$ is $C^{1}$ on $U_{1} \times U_{2}$, and $f_{1}, f_{2}$ are $C^{1}$ on $U$.

Since $c, b$ are independent generic elements in $I^{n},(c, b)$ is a generic element in $I^{n} \times I^{n}$, and, by Fact 1.1, we can find such $U_{1}, U_{2}$.

Since $f_{1}$ is definable over $b$ and $a$ is generic over $b$, there is open $U^{1}$ containing $a$ such that $f_{1}$ is $C_{1}$ on $U^{1}$. By the same reason, there is open $U^{2}$ containing $a$ such that $f_{2}$ is $C_{1}$ on $U^{2}$. We can take $U=U^{1} \cap U^{2}$.

Let $V$ and $\varphi$ be as in Claim 3.12. Identifying $V$ with $\varphi(V)$ we can assume $\varphi=$ id.

Claim 3.13. If $h$ is a definable endomorphism of $G$, then there is open $D \subseteq V$ containing e such that $h(D) \subseteq V$ and $h$ is $C^{1}$ on $D$.

Proof. By Lemma 1.11, there is open $U$ containing e such that $h(U) \subseteq V$.

By Fact 1.1, $h$ is $C^{1}$ on some open $U_{0} \subseteq U$. Take any $a \in U_{0}$, and let $D=$ $a^{-1} \star U_{0}$. Since the group operations are $C^{1}$ on $V$, using the Chain Rule, it is easy to show that $h$ is $C^{1}$ on $D$.

By the previous claim, if $h$ is a definable automorphism of $G$, then the differential of $h$ at $\mathbf{e}$ (in the sense of $\mathcal{R}$ ) exists.

Claim 3.14. Let $f_{1}, f_{2}$ be two definable automorphisms of $\mathbb{G}$ such that the differential of $f_{1}$ at $\mathbf{e}$ is equal to the differential $f_{2}$ at $\mathbf{e}$. Then the definable subgroup $H=\left\{g \in G \mid f_{1}(g)=f_{2}(g)\right\}$ has finite index in $\mathbb{G}$.

Proof. By the same arguments as in the proof of Lemma 3.2 (ii) in [7], $H$ contains an open neighborhood of $\mathbf{e}$, so it has finite index in $G$.

Now we can finish the proof of Theorem 3.2 ,

For each $g \in G$ consider the inner automorphism

$$
\operatorname{Int}_{g}: x \mapsto g \star x \star g^{-1} .
$$

Let $\operatorname{Ad}(g)$ be the differential of $\operatorname{Int}_{g}$ at $\mathbf{e}$. Then Ad is a definable homomorphism from $G$ into $\operatorname{GL}(n, \mathcal{R})$, and, since $\mathbb{G}$ is $\mathbb{G}$-definably connected and centerless, by Claim 3.14 it is one-to-one.

\section{The MAIN Result}

Let $G$ be an infinite group definable in an o-minimal structure $\mathcal{M}$. The main result of this paper is:

Theorem 4.1. Assume $G$ is $\mathbb{G}$-definably connected and has no nontrivial abelian normal subgroup. $G$ is the direct product of $\mathbb{G}$-definable subgroups $H_{1}, \ldots, H_{k}$ such that for every $i \in\{1, \ldots, k\}$ there is a definable real closed field $\mathcal{R}_{i}$ and a definable isomorphism between $H_{i}$ and a semialgebraic subgroup of $\mathrm{GL}\left(n_{i}, \mathcal{R}_{i}\right)$. Every $H_{i}$ is $\mathbb{H}_{i}$-definably simple and its $\mathcal{M}$-definably connected component $H_{i}^{0}$ is definably simple.

Remark 4.2. Although it is true that every $H_{i}$ above is $\mathcal{M}$-definably connected, the only way to show it, that we know, is through careful analysis of such groups that will appear in [9]. It will require, in particular, the next corollary that follows from Theorems 4.1 and 2.36 .

Corollary 4.3. Let $G$ be a $\mathbb{G}$-definably simple group definable in an o-minimal expansion of a real closed field. Then the Lie algebra of $G$ is simple. 
The following corollary can be viewed as an o-minimal analogue of Cherlin's conjecture.

Corollary 4.4. Let $G$ be a $\mathbb{G}$-definably simple group definable in o-minimal structure $\mathcal{M}$. Then there is a real closed field $\mathcal{R}$ definable in $\mathcal{M}$ such that $G$ is definably isomorphic to a semialgebraic linear group over $\mathcal{R}$.

Proof of Theorem 4.1. By Theorems 3.1 and 3.2, $G$ is the direct product of $\mathbb{G}$ definable subgroups $G_{1}, \ldots, G_{m}$ such that for every $i \in\{1, \ldots, m\}$ there is a definable real closed field $\mathcal{R}_{i}$ and a definable isomorphism $f_{i}$ between $G_{i}$ and a subgroup of GL $\left(n_{i}, \mathcal{R}_{i}\right)$. Since every $G_{i}$ is $\mathbb{G}$-definable, it is easy to see that every $G_{i}$ is $\mathbb{G}_{i}$-definably connected and has no nontrivial abelian normal subgroup.

Let $G_{i}^{\prime}=f_{i}\left(G_{i}\right)$. Then $G_{i}^{\prime}$ is definable in an o-minimal expansion of a real closed field $\mathcal{R}_{i}$, and, since $G_{i}^{\prime}$ is isomorphic to $G_{i}, G_{i}^{\prime}$ is $\mathbb{G}_{i}^{\prime}$-definably simple and has no abelian nontrivial normal subgroup. Obviously, it is sufficient to prove the theorem for each $G_{i}^{\prime}$. Thus we will assume that $\mathcal{M}$ is an o-minimal expansion of a real closed field $\mathcal{R}$.

Let $G^{0}$ be the definably connected component of $G$.

Claim 4.5. $G^{0}$ has no nontrivial abelian normal subgroup and therefore is semisimple.

Proof. Suppose $A$ is an abelian normal subgroup of $G^{0}$. Let $K=\mathbf{C}_{G}(A)$ and let $H$ be the center of $K$. Obviously, $A<H$ and, by Corollary 1.16, both $K$ and $H$ are $\mathbb{G}$-definable. Consider $\mathbf{N}_{G}(H)=\left\{g \in G \mid H^{g}=H\right\}$. Since $A$ is a normal subgroup of $G^{0}, \mathbf{N}_{G}(H)$ contains $G^{0}$ and, as $G$ is $\mathbb{G}$-definably connected, $\mathbf{N}_{G}(H)=G$. Hence $H$ is a normal abelian subgroup of $G$, so $H=\{\mathbf{e}\}$ and $A=\{\mathbf{e}\}$.

By the previous claim and Theorem 2.37, $G$ is definably isomorphic to a linear semialgebraic group over $\mathcal{R}$. Thus, without loss of generality, we can assume that $G$ is linear and semialgebraic.

The following claim and an easy induction on $\operatorname{dim}(G)$ finish the proof of the theorem.

Claim 4.6. If $G^{0}$ is not definably simple, then $G$ is the direct product of nontrivial $\mathbb{G}$-definable subgroups.

Proof. Assume $G^{0}$ is not definably simple. By Theorem 2.38, $G^{0}$ is the direct product of definable nontrivial subgroups $K_{1}$ and $K_{2}$. Let $G_{1}=\mathbf{C}_{G}\left(K_{2}\right)$ and $G_{2}=\mathbf{C}_{G}\left(K_{1}\right)$. It is easy to see that both $G_{1}$ and $G_{2}$ are $\mathbb{G}$-definable, they commute, $K_{1}<G_{1}$ and $K_{2}<G_{2}$.

The subgroup $G_{1} \star G_{2}$ is $\mathbb{G}$-definable and contains $G^{0}$, so it is equal to $G$. Since $G$ is centerless, $G_{1} \cap G_{2}=\{\mathbf{e}\}$ and $G$ is the direct product of $G_{1}$ and $G_{2}$.

\section{REFERENCES}

[1] L. van den Dries, Tame topology and o-minimal structures, London Math. Soc. Lecture Note Ser., vol. 248, Cambridge Univ. Press, Cambridge, 1998. MR 99j:03001.

[2] L. van den Dries, C. Miller, Geometric Categories and o-minimal structures, Duke Math. J. 84(1996), pp. 497-540. MR 97i:32008

[3] J. E. Humphreys, Introduction to Lie Algebras and Representation Theory, Springer-Verlag, 1972. MR 48:2197]

[4] J. Knight, A. Pillay and C. Steinhorn, Definable sets in ordered structures II, Transactions of the Amer. Math. Soc. 295 (1986), pp. 593-605. MR 88b:03050b 
[5] J. Loveys and Y. Peterzil, Linear o-minimal structures, Israel J. Math. 81 (1993), pp. 1-30. MR 94i:03075

[6] A. L. Onishchik, E. B. Vinberg, Lie Groups and Algebraic Groups, Springer-Verlag, 1990. MR 91g:22001

[7] M. Otero, Y. Peterzil and A. Pillay Groups and rings definable in o-minimal expansions of real closed fields, Bull. London Math. Soc. 28 (1996), pp. 7-14. MR 96i:12006

[8] A. Pillay and C. Steinhorn, Definable sets in ordered structures I, Transactions of the Amer. Math. Soc. 295 (1986), pp. 565-592. MR 88b:03059

[9] Y. Peterzil, A. Pillay and S. Starchenko, Simple algebraic groups over real closed fields, Trans. Amer. Math. Soc. 352 (2000), 4421-4450.

[10] Y. Peterzil and S. Starchenko, A trichotomy theorem for o-minimal structures, Proc. London Math. Soc. 77 (1998), pp. 481-523. CMP 99:01

[11] A. Pillay, On groups and fields definable in o-minimal structures, Journal of Pure Applied Algebra 53 (1988), pp. 239-255. MR 89i:03069

[12] B. Poizat, Groupes Stables, Nur al-Mantiq Wal-Ma'rifah, 1987. MR 89b:03056

[13] A. W. Strzebonski, Euler characteristic in semialgebraic and other o-minimal groups, J. Pure Appl. Algebra 96 (1994), no. 2, pp. 173-201. MR 95j:03067

Department of Mathematics and Computer Science, Haifa University, Haifa, Israel

E-mail address: kobi@mathcs2.haifa.ac.il

Department of Mathemetics, University of Illinois at Urbana-Champaign, 1409 W. Green St., Urbana, Illinois 61801

E-mail address: pillay@math.uiuc.edu

Department of Mathemetics, University of Notre Dame, Room 370, CCMB, Notre DAme, Indiana 46556

E-mail address: starchenko.1@nd.edu 